\title{
Neutron Sawtooth Behavior in the PLT, DIII-D, and TFTR Tokamaks
}

\author{
J.A. Lovberg, $\quad$ W.W. Heidbrink, ${ }^{\dagger} \quad$ J.D. Strachan, \\ V.S. Zaveryaevt \\ Plasma Physics Laboratory, Princeton University, \\ Princeton, New Jersey, USA
}

\begin{abstract}
The effect of the sawtooth instability on the $2.5 \mathrm{MeV}$ neutror emission in the PLT, DIII-D, and TFTR tolamaks is studied. In thermonuclear plasmas, the instability typically results in a $20 \%$ reduction in errission. The time evolution of the thermonuclear neutron signal suggests that the sawtooth crash consists of four phases. First, the electron density profile flattens rapidly (in $\sim 30 \mu$ sec on PLT) but, in sone cases, there is little asociated change in neutron emission, sugsesting that most reacting ions remsin confined in the sawtooth region but do not completely mix. After the electron sawtooth, the ions continue to mix, resulting in a 10\% reduction in neutron emission in $\sim 0.5$ meec. The emission then decays more slowly during the final two phases. Thermalization of reacting ions on a $\sim 3 T_{i i}$ time scale accounts for only $\sim 20 \%$ of the slow drop. Most of the slow drop seems to be caused by loss of ion energy from the mixing region (an ion heat pulse).
\end{abstract}

\footnotetext{
"Present addren: Maxwell Luboratories, Lac., San Diego, Califormin, USA

'Present eddrea: Department of Physies, University of Califomian Irvine, Califomia, USA

'Permanent addrew: I.V. Kurchrtov Inetitute of Atomic Energy, Moscow, USSR
} 


\section{INTRODUCTION}

An understanding of the effect of the sawtooth instability on ions is important in studies of ion transport and in calculations of the performance of proposed ignition experiments. ${ }^{2}$ Sawtooth behavior in the $2.45 \mathrm{MeV}$ neutron emission was observed previously on the Alcator-A, ${ }^{2}$ TFR, ${ }^{3}$ and $\mathrm{JET}^{4}$ tokamaks and, more recently, in collimated neutron measurements on JET. ${ }^{5}$ Sawteeth in the central ion temperature have been measured with $\mathrm{X}$. ray spectroscopy on TFTR $^{\circ}$ and with charge-exchange recombination spectroscopy on PBX. ${ }^{\top}$ On PBX, time-resolved measurements showed that the ion temperature profile flattens at the sawtooth. ${ }^{\top}$ Flattening of the density profiles of impurity ions at sawteeth was observed on Alcator $C .^{8}$

In our work, the temporal resolution $(210 \mathrm{kHz})$ of the neutron measurements was faster then the observed decay rates, permitting a more detailed study of the temporal behavior of ions at sawteeth than was possible in earlier studies. The paper has two main aims. One is to catalog the effect of sawteeth on the neutron source strength in tolcarnaks. Thermonuclear neutron data from the PLT, DIII-D, and TFTR tokamaks have been obtained with similar instrumentation under a wide range of operating conditions in plasmas with ohmic heating alone, hydrogen neutral beam injection into L-mode and H-mode plasmas, ICHH heating, and pellet injection. In a wide variety of plasmas, similar behavior is observed. Generally, a rapid initial drop in emission is observed followed by a slower decay. The initial drop in emission often take longer than the electron temperature sawtooth. The time scale of the alower drop depends on machine size, varying approximately as the minor radius squared $\left(a^{2}\right)$. The total drop in neutron emission $\Delta I_{n} / I_{n}$ is usually larger than the reduction in central electron temperature $\Delta T_{e} / T_{e}$.

The second major sim in to explain the neutron signal by considering possible ion behavior at the sumtooth. Four proceses are identified. First, on the time scale of the electron sawtooth, the ion density must flatten with the electron denuity to preserve quacineutrality; however, the neutron emiecion dropa gradually a though hot deuterons respond more slowly than electrons. Second, after the electron sawtooth, the icn velocity-space distribution is blended in an "ion mixing" phase and the emission falls $\sim 10 \%$. Generally, the density crash and mixing phases are too short for significant classical energy loss by the reacting ions. In the third phase, the hot ions that produce the reactions begin to thermalize, but the time scale is suf- 
ficiently long that, in some plasmas, a Maxwellian distribution may never be established. Concurrently, in the fourth process, the plasma responds to the flattened density and "temperature" profiles. At the edge of the mixing region, a steep gradient exists and rapid transport occurs. Both thermalization and diffusion result in a further $\sim 10 \%$ reduction in emission on a 10 msec time scale. In our analysis, the "transport" and "thermalization" phases terminate when the geutron emission ceases to drop as the plasma reheats and the density peaks.

The paper is organized as follows. The temporal resolution of the detectors and the plasma conditions are discussed in Sec. II. Thermonuclear neutron data from PLT, DIII-D, and TFTR are presented in Sec. III. Next, the expected behavior during the elestron crash phuse (Sec. IVA), mixing phase (Sec. IVB), thermalization phase (Sec. IVC), and transport phase (Sec. IVD) is compared with the data. We conclude (Sec. V) that 1) hot ions are not mixed uniforaly throughout the sawtooth region during the electron crash phase but may be mixed on a longer time scale, 2) thermalization probably does not play a dominant role in the observed tempcral evolution, and 3) an ion heat pulse escapes from the center of the plamis. Finally, in two appendices, it is shom that neutron and $15 \mathrm{MeV}$ proton data from beam-plarms resctions are consistent with our interpretation of the thermonuclear data.

\section{APPARATUS}

\section{A. Neutron Diagnostirs}

Fast neutron detectors 9.10 that utilize uncollimated plastic scintillators mounied close to the tokmak were the primary diagonotics for this study. Similar detectors were used on all three machines. Neutrons strike a plartic scintillator producing recoil protons that cause the plestic to scintillate. The lipht travels by internal reflection down an acrylic rod to a shielded photomultiplier operated in current mode. The photomultiplier signal is digitized by a trantient digitizer. Geterally, thre factors limit the temporal response of the detectors: counting statistics, amplifier/digitizer frequency response, and neutron scattering. The intrinsic response of the plastic scintillator is $<0.1$ psec. However, at low levels of neutron emission, the signal-to-noise ratio is degraded by statistical fluctuations in the number of neutrons that collide with the scintillator, which results in poorer temporal resolution. Also, on TFTR, electrical noise placed a lower limit on the useful signal 
level. When an adequate signal existed, the frequency response of the detectors was limited by the photomultiplier amplifier to $\sim 20 \mathrm{kHz}$ on PLT and TFTR and to $\sim 100 \mathrm{kHz}$ on DIII-D. The digitizer sampling rate was $100 \mathrm{kHz}$ on FLT and DII-D and $20 \mathrm{kHz}$ on TFTR.

The frequency response of the detectors was nominally $\sim 20 \mathrm{kHz}$ but there is an additional factor that could degrade the resolution further: neutron scattering. If detected in appreciable numbers, neutrons that bounce around the tolamak hall could significantly degrade the frequency response. To minimize this effect, the detectors were placed cloee to the machine between toroidal field coils. On TFTR, the scintillator was mounted directly behind a $1.3 \mathrm{~cm}$ thick stainless steel vacuum flange at the horizontal mid. plane. On DIII-D, the scintillator was mounted normal to a $2.5 \mathrm{~cm}$ thick stainless steel flange that is $85 \mathrm{~cm}$ sbove the horizontal midplane. Similar remults to the ones reported here were obtained with the scintillatar on the horizontal midplane. On PLT, the scintillator was mounted $\sim 1 \mathrm{~m}$ away from the vacuum vessel near the midplane. Each of these installa tions has a different gecmetry for neutron scattering. Detailed scattering calculations have not been performed but it is thought that seattering does not significantly degrade the frequency response since neutron transients as rapid as $\dot{I}_{n} / I_{n}=0.8$ msec $^{-1}$ (which is $\sim 4$ times faster than the transients reported here) have been observed during high $\beta$ instabilities. ${ }^{11,12}$ Also, when the plasma center moved $83 \mathrm{~cm}$ during compression on TFTR ${ }^{13}$ and $\sim 20 \mathrm{con}$ during the fishbone intability on PDX, ${ }^{11}$ the magnitude of the neutron emisuion changed as expected for a virgon (unucattered) source. These observations suggest that neutren scattering does not limit the frequency response of our meaturements.

On all three tolomakn, neutron measurements with the platic scintillators were corroborated by slower mearurements ( $\sim .2$ meec resolution) made with adjacent $\mathrm{ZnS}\left({ }^{\circ} \mathrm{L}\right)$ scintillators. ${ }^{10}$ The mearurements also were consistent with meacurements made with $\mathrm{BF}_{3}$ proportional counters (PLT and DIII-D) and firsion detectors (TFTR).

\section{B. Tokamak Conditions}

The PLT sawtooth observations used in this study were made during a series of high-power ${ }^{3}$ He-minority ICRH runs in late 1984 and early 1985. Discharges in the database have the parameter ranges $I_{p}=500-600 \mathrm{kA}$, $B_{z}=31-33 \mathrm{kG}, \bar{n}_{2}=2-4 \times 10^{13} \mathrm{~cm}^{-3}$, and $T_{\infty 0}=2.0-3.2 \mathrm{keV}$. In addi- 
tion to neutron data, the database includes electron temperature and density profiles from Thomson scattering, $Z_{\text {aff }}$ inferred from a sentral-chord visible-bremsstrahlung mearurement, and fractional changes in central electron temperature and line-averaged electron density at the sawtooth from electron cyelotron emission and microwave interferometry. Additional observations on PLT were made in $H^{0} \rightarrow D^{+}$neutral-beam-heated plasmas, in H-minority ICRH-heated deuterium plasman, and in ohmically heated deucerium plasmas.

The TFTR obervations were made during the Spring 1985 mu period and include sawteeth in full size $\left(a \simeq 81 \mathrm{~cm} ; R_{0} \simeq 255 \mathrm{~cm}\right)$ plasmas with $I_{p}=0.8-2.2 \mathrm{MA}, B_{t}=4.0-4.8 \mathrm{~T}, \bar{n}_{0}=2-6 \times 10^{13} \mathrm{~cm}^{-3}$, and $T_{c}(0)=1.8-$ $3.5 \mathrm{keV}$. Some of the plems were pellet fuelied and some were gat fuelled. About 70 sawteeth for which concurrent Thomscon scattering data and soft $X$-ray (SXR) measurements of the sawtooth inversion radius were available were selected for inclusion in a databare. Subordinate relaxations ${ }^{14}$ were excluded from the study al though they were oberved to correlate with small ( 2\%) reductions in neutron emiazion. In addition to neutron data, the databace included $\boldsymbol{Z}_{\text {eff }}$ inferred from a central visible bremestrahlung chord, the fractional change in central SXR emiscion $\Delta A / A$ and central electron temperature $\Delta T_{.} / T_{4}$ (from ECE), the magnitude of and fractional change in line-averaged density $\Delta \bar{n}_{*} / \bar{n}_{0}$, the shape of the electron temperature and density profiles (from Thomson scattering), and the central ion temperature inferred from Dopplex broadening of the $T i K_{a}$ line.

Data from DIII-D were obthined in 1987 during $H^{0} \rightarrow D^{+}$beam injection over the parameter range $I_{p}=0.7-1.8 \mathrm{MA}, B_{t}=1.0-2.1 \mathrm{~T}$, $\bar{n}_{n}=3-12 \times 10^{13} \mathrm{~cm}^{-3}$, and $R \leqslant 10 \mathrm{MW}$. Mont of the plasmas were divertor discharges and many were $\mathbf{H}$-mode ${ }^{13}$ placmas. The reduction in central electron temperature at a s2wtooth was meanured using ECE.

An assumption of our analysis is that the neutron emission is thermonuclear. For ohmic, $H^{0} \rightarrow D^{+}$beam, and ${ }^{3} \mathrm{He}$ or $\mathrm{H}$ minority ICRH heating, the deuterium population in thought to be heated only through collisions with electron, energetic protons, or energetic ${ }^{3} \mathrm{He}$ ions, so the deuterium distribution is erpected to be nearly Marwellian. Central ion temperatures inferred from charge-exchange spectra and Doppler brosdening of impurity lines are generally consistent with the temperature inferred from the neutron flux assuming thermonuclear emission. ${ }^{16}$ The charge-exchange spectra ${ }^{17}$ appear Maxwellian out to energies of $\sim 10 T_{d}$. Further confirmation of the thermonuclear origin of the emission comes from collimated neutron spectral 
measurements, which indicate no nonthermal broadening or shifting of the $2.45 \mathrm{MeV}$ neutron emission line during $\mathrm{H}^{0} \rightarrow D^{+}$neutral beam injection on PLT, ${ }^{\text {16 }}{ }^{3} \mathrm{He}$-minority ICRH heating on PLT, ${ }^{19}$ or ohmic heating on $\mathrm{TFTR}^{20}$ or PIT.

\section{DATA}

Typical neutron suwtooth behavior during ${ }^{3} \mathrm{He}$-minrsity ICRH heating in PLT is shown in Fig. la. No significsnt change $(<1 \%)$ in neutron emission accompanies the large, sudden drop is electron temperature (typically $25 \%$ in $50 \mu \mathrm{\mu ec}$ ) seen at the sawtooth crah (Fig. 2). Subrequently, the neutron emisnion drope an a slower time ecale ( $\sim 10 \%$ reduction in $\sim 0.5 \mathrm{msec}$ ) and then declines an additional $\sim 5 \%$ at a still slower rate to an absolute minimum in about 5 mrec. This change in the rate of decay of the emission is seen more clearly by plotting the slope of the neutron signal (Fig. 3). The overall reduction in emiscion at this swtooth was $\Delta I_{n} / I_{n}=18 \%$. After the minimum, the eminion increaces until the mbsequeat sawtooth occurs $\sim 15$ msec later. The ion temperature was $T_{2} \simeq 3 \mathrm{keV}$ in this diacharge.

Neutrod sawtooth behavior during obmic heating in TFTR is illustrated in Fig. $1 \mathrm{~b}$ from a plame fuelled by pellets at 1.8 and $2.1 \sec \left(T_{d} \simeq 2.0 \mathrm{keV}\right)$. The drop in neutron emiscion $\left(\Delta I_{n} / I_{n}=22 \%\right)$ is comparable to that observed in PLT but the signal takes approximately four times longer to reach its minimum. The initial rate of decay of the emiscion is comparable for the two devices, even though the electron temperature drop occurs more slowly (100 peec) ;n TFTR than in PLT. In both machines, there appears to be a discontinuity in the rate of decay of the emistion $\sim 0.5$ msec after the sawtooth. The sawtooth period in this TFTR plames (not shown) is $\sim 8$ times longer than in the PLT plasma.

A neutron sawtooth during the H-mode in DIII-D is shown in Fig. 4. The initial rate of decay of the neutron emission is similar to that observed on PLT and TFTR. The signal reaches its minimum value faster than a typical TFTR sawtooth but lower than a typical PLT sawtooth. The sawtooth period $(\sim 50 \mathrm{~ms})$ is also intermediate between PLT and TFTR. The overall drop in emission was $\Delta I_{n} / I_{n}=17 \%$ at this sawtooth in a plama with $T_{d} \simeq 1.3 \mathrm{keV}$. Typical sawtooth behavior in the three devices is compiled in Table 1.

Several features of the initial drop in emission are noteworthy. One striking feature of the data is that the initial rate of decay is comparable 
on the three devices (Table 1). In contrast, the electron crash time, the overall neutron decay time, and the sowtooth period differ greasly between machines. A second feature is that, in some plarmas, little drop in neutron emission occurs on the time scale of the electron sawtooth. This is seen most clearly on PLT (Fig. 2), where the electron sawtooth is fastest, but is also observed at some sawteeth on DIII-D (Fig. 5). A third feature of the initial decay is its variability. The decay rate at nominally similar sawteeth in the same plasma varies by nearly a fuctor of two under some DIII-D and TFTR conditions. In light of this variability, it seems unlikely that the initial rate of decay depende on global discharge parameters in a imple manner. Indeed, little correlation with $\bar{n}_{n}, T_{n}$, or $T_{i}$ is observed. On PLT, the magnitude of the initial drop carrelated with the fractional change in electron temperature and with the eawtooth radiu; this correlation was not oberved in DIII-D, however. In summary, the initinl decay of the emincion is 1) comparable in different tokamniks, 2) sometimes alower than the electron sawtooth, and 3) variable in its dependence con glohal parametere.

After the initial rapid decay, the emincion fills gradually to a minimum. For PLI, the peak-to-trough sawtooth time $t,-t$ scales with the magnitude of the sawtooth period (Fig. 6), which aleo scales with the magnitude of the neutron drop $\Delta I_{n} / I_{n}$, but this sealing res not obeerved on TFTR. No direct correlation with heating method or porar, electron temperature $\alpha$ density, central ion temperature, toroidal migotic field, or plame current was observed in any device. A comparison of $\zeta_{-2}$ for different devicen (Fig. 7) indicates that the pesk-to-trough time scale approminately with machine minor radiu to the second power $\left(a^{2}\right)$, sugesting a dependence on transport processes. A quantitative comparison of the PLT duta with a transport model is given in Sec. $D$.

For a given discharge condition, the drop in neutron emierion from peakto-trigh $\Delta I_{n} / I_{n}$ scale approximately linearly with the drop in electron temperature $\Delta T_{e} / T_{e}$ in all three tokardaks. This is illustrated in Fig. 8 for ohmically heated TFTR plesmas. For these plarmus with $T_{d} \simeq 2.0 \mathrm{keV}$, $\Delta I_{n} / I_{n} \simeq 1.6 \Delta T_{i} / T_{c}$. In ICRE-heated PLT plarmw a linear correlation is also observed bul, in these hotter playman $\left(T_{d} \simeq 3.0 \mathrm{keV}\right)$ the coefficient of proportionality is smaller $\left(\Delta T_{n} / I_{n} \simeq 0.6 \Delta T_{c} / T_{c}\right)$. In $H^{0} \rightarrow \Sigma^{+}$, DIII-D H-mode plasmas, the ion tempereture is lower than in TFTR ( $T_{d} \simeq 1.3 \mathrm{keV}$ ) and the scaling of $\Delta I_{n} / I_{n}$ with $\Delta T_{d} / T_{c}$ is stronger $\left(\Delta I_{n} / I_{n} \simeq 2.1 \Delta T_{c} / T_{e}\right)$. Presumably, some of the variation in the coefficient of proportionality is due to the temperature dependence of the fusion reactivity. For $\overline{\sigma v} \times T_{d}^{c}$, where 
$c$ is a function of temperature (and assuming that the ion temperature sawtooth is responsible for most of the drop in neutron emission), the expected reduction in aeutron emission is roughly $\Delta T_{n} / I_{n} \simeq c \Delta T_{d} / T_{d}$. This implies that $\Delta T_{d} / T_{d} \simeq 0.4 \Delta T_{d} / T_{e}$ in DIII-D and TFTR but $\Delta T_{d} / T_{1} \simeq 0.2 \Delta T_{e} / T_{\text {. }}$ in PLT. Thus, the temperature dependence of the reactivity accounts for some of the variation in scaling between devices but cannot fully explain the differences. One possibility is that the drop in neutron emission is smaller on PLT because the wawtooth period is shorter so that the plasma begins to reheat prior to termination of the transport phase. On PLT, the initial drop $\left(\delta I_{n}\right.$ after $\left.\sim 0.5 \mathrm{msec}\right)$ is a larger fraction of the total drop $(\simeq 2 / 3)$ than on TFTR $(\simeq 1 / 3)$ or on DIII-D $(\simeq 1 / 4)$ (Table 1 ).

\section{ANALYSIS}

The behavior of the thermomuclear neutrop eminion at a sawtooth suggests that several physical processes are responsible for the observed time evolution. In this section, we conxides four phuses in the evolution of the ion distribution function in an attempt to explain our results. The phates are illustrated in Fig. 9. In the first phace (Fig. 9a, Sec. A), the ion density flatens to preseve quasineutrality when the electron density collapses. At the end of this phase, the deuterium number density is uniform within the mixing radius but the velocity ditribution is not. This phese results in little change in seutron emistion. In the second phase (Fig. 9b, Ssc. B), the velocity distribution becomes homogeneous and the emisrion falle. At the end of thin phase, the velocity distribution is not Marmellian but it is uniform in space. The last two phases tun concurrently: the perturbed ion distribution relaxes to $=$ Maxwellien distribution (Fig. 9c, Sec. C) and an ion heat pulse proparstes away from the plamm center the steep gradient in the ion temperature new the mixing radius diffures (Fig. 9d, Sec. D). Both of these procestes cause further reduction in the neutron tate. The aeutron sawtooth ends when ion heating overcomes the energy los from thermal conduction and the central deuterium density begins to peak on aris. When the pre-wawtooth conditions are reestablished, the cycle is repeated. In the following four subections, the predieted effect of each of these processes is derived and compared with experiment. 


\section{A. Density Crash Phase}

The sawtooth instability has been observed to flatten (or slightly hollow) the electron density on $\mathrm{TFR}^{3}$ and sawtooth behavior in $n_{\text {- }}$ has been clearly observed on TEXT, ${ }^{21}$ PLT Thomson-scattering and TETR five-channel incerferometer ${ }^{6}$ mearurements are consistent with these observations. Since the plasma is constrained to remain quasineutral, the electron density crash implies a concurrent ion density crash. Measurements on Alcator $\mathrm{C}^{\mathbf{b}}$ indicate that the inpurity silicon density is flattened at a sawtooth. While it is possible that the density sawtooth for deuterons cculd differ from that for impurity ions, observations with $\mathrm{d}\left({ }^{3} \mathrm{He}, \mathrm{p}\right)$ a proton detectors reported here in low $Z_{\text {aff }}$ discharges give direct evidence of deuterium deasity .iatten. ing on the time scale of the electron denuity sawtooth cranb (Appendix B). Previous experiments with d. ${ }^{3}$ He proton detectors on PLT ${ }^{22}$ and JET ${ }^{20}$ and active tharge-exchange ${ }^{24}$ on PLT ruggest that energetic ${ }^{3} \mathrm{Hl}$ ions and fast denterons are also redistributed at a sutooth.

Canceivably, deuterons could gain energy during the craeh phase, as was observed during dimuptions on LT-3. ${ }^{26}$ This would result in an increve in neutron emiscion. Alternatively, if a significant fraction of hot tail deuterons were redistributed over much of the ph me, the emistion would be expected to drop. Experimentally on PLT, little change $(<1 \%)$ is observed in the neutron emivion on the time scale of the electron temperature watooth crach (Fig. 2). Typieally, a $\sim$ 4\% drop in $I_{n}$ is observed an DIII-D and TFTR during the electron crah phue, but some of this drop may be arsociated with the miring phese, since the electron surtooth is slower then on PLT. The frequency response of the neutron system on PLT is suffcient to record fluctuations three to four times faster then those observed in Fig. 2, so it appears that the neutron race is acturlly quite insensitive to the electron redistribution. Neverthelea, $d\left({ }^{3} \mathrm{He}, \mathrm{p}\right)$ a proton mearurements show that the deuterium denvity profile is redistributed on the fast ( $30 \mu \mathrm{\mu s})$ time tcale (Appendix B). As shown in the next subsection (Sec. B), if the ions were uniformly mixed at the electron sawtootb, a $~ 5 \%$ reduction in neutran emission is expected. Conceivably, some liberated magnetic energy could hent the icar, thereby making the drop weociated with ion mixing. This explanntion seerns unlikely, however, since the peutron emition does drop to the expected level on a 1 meec time scale and it seems unlikely that this liberated energy would be so rapidly lost. How then ean the density fiatten without the ions mixing? One postibility in that the ion denaity flattening is accomplished locally through small ( $\leqslant 0.2 \mathrm{~cm}$ ) displacements of 
bulk-plasma volume elements (Fig. 10); such a redistribution would have a small effect on the volume-integrated fusion emission. This is analogous to the way that a mound of viscous jelly would flatten under the influence of gravity; little real particle mixing would be involved. A second possibility is that the deuterons collapse like a mound of pebbles: in this scenario, the density after the crash is fairly uniform but fine-scale variations in temperature still exist. A third possibility is that the warm, fusion-producing ions do not mix as rapidly as the thermal ions.

In summary, the relatively small drop in neutron emission on the time scale of the PLT sawtooth suggests that 1) little energy is lost or gained by the ions at the crash and 2) the velocity distribution function $f(r, v)$ retains some dependence on $r$ after the crash. In the next phase, the "mixing phase", this spatial structure is lost and the velocity distribution becomes uniform.

\section{B. Mixing Phase}

A. drop in neutron emission is expected if the ion temperature and density are flattened at a sawtooth. In this subsection, we calculate the expected reduction in emission for a uniform redistribution within the mixing radius using a heuristic analysis (Sec. 1). Next, the predicted drop is calculated numerically (Sec. 2) and compared with experiment (Sec. 3).

\section{Heuristic Analysis}

The $d(d, n)^{3}$ He neutron emission rate in a deuterium plasma is

$$
I_{n}=\int \frac{1}{2} n_{d}^{2}(\sigma v)_{d, n} d V,
$$

where $n_{d}$ is the deuterium density and $(\sigma v\rangle_{\alpha, n}$ is the $d(d, n)^{3} \mathrm{He}$ fusion reactivity

$$
\langle\sigma\rangle_{d n} \equiv \iint f_{1}\left(v_{1}\right) f_{2}\left(v_{2}\right) \sigma v d v_{1} d v_{2} \text {. }
$$

Here $v_{1}$ and $v_{2}$ are the velocities of the reacting particles, $v=\left|v_{1}-v_{2}\right|$, and $\sigma$ is the $d(d, n)^{3}$ He fusion cross section. For a Marwellian plasma of temperature $T_{d}$, this equation can be rewritten in terms of the center-ofmass velocity $V_{o n}$ and relative velocity $v^{20}$ :

$$
\langle\sigma v\rangle_{d, n}=\left\{\left(\frac{m_{d}}{4 \pi T_{d}}\right)^{3 / 2} \int \exp \left(-\frac{m_{d} v^{2}}{4 T_{d}}\right) \sigma(v) v d v\right\} \times
$$




$$
x\left\{\left(\frac{m_{d}}{\pi T_{d}}\right)^{3 / 2} \int \exp \left(-\frac{m_{d d} V_{m m}^{2}}{T_{d}}\right) d V_{c m}\right\} .
$$

Using the Gamow cross-section of form $\sigma(v)=\left(C_{1} / v^{2}\right) \exp \left(-C_{0} / v\right),{ }^{27}$ the integrand of the relative velocity integral has a sharp maximum for $\left(C_{0} v_{t h}^{2}\right)^{-1 / 3} v=$ 1 , with $C_{0}=1.482 \times 10^{\circ} \mathrm{cm} / \mathrm{sec}$. This corresponds to $v / v_{t h}=3.63 T_{d}^{-1 / 0}$, for $T_{d}$ in $\mathrm{keV}$. The integrand of the center-of-mass velocity intagral is much broader, giving a mont probable value of $V_{a m} / v_{t h}$ at $1 / \sqrt{2}$ and an average value at $\sqrt{2 / \pi}$. Thus the ratio of relative to center-of-mass velocities of the deuterons invalved in a typical fusion reaction is $v / V_{o n} \simeq 4.3$ for $T_{d}=3$ $\mathrm{keV}$, which implies that the mont probable reaction is one between ions in the tail of the distribution directed in nearly opposite directions. A typical reacting ion has energy

$$
E_{h}=\frac{1}{2} m_{d} v_{1}^{2} \simeq \frac{1}{2} m_{4}\left[V_{m}^{2}+\left(\frac{v}{2}\right)^{2}\right] \simeq\left[\frac{1}{2}+\frac{1}{4}\left(\frac{C_{0}}{v_{t h}}\right)^{2 / 3}\right] T_{d}
$$

which implies $E_{h} \simeq 3 T_{d}$ for ion temperatures near $3 \mathrm{keV}$.

The effect on the neutron emiscion of deuterium mixing can now be treated with a simple heuristic model. In this model, the deuterium particle and velocity distributions are made uniform inside of a mixing radius $r_{\text {st }}$ at the sawtooth craah. In the mixing region, $I_{n} \simeq k \int_{0}^{\mu t} n_{h}^{2} r d r$, where $n_{h}$ is the density of hot ions with $E_{h} \simeq 3 T_{d 0}$ that produce most of the fuxion reaction, and $h$ in a constant proportional to the $\mathrm{d}(\mathrm{d}, n)^{3} \mathrm{He}$ fusion reactivity. Prior to the sawtooth eranh, $n_{h} \equiv \vec{n}_{h}+\delta n_{h}$, where $\bar{n}_{h}$ is the post-crash density and $\int_{0}^{r} \delta n_{h} r d r=0$ by particle conservation. After mixing, the emission in the sawtooth region is

$$
I_{a, r<r_{11}}=k \int_{0}^{m} \bar{n}_{h}^{2} r d r,
$$

while beforehand, the emission in the same region is

$$
\begin{aligned}
-I_{b_{1},<r_{n t}} & =k \int_{0}^{r a t}\left(\bar{n}_{h}+\delta n_{h}\right)^{2} r d r \\
& =I_{a_{1}, r<r_{n t}}+k \int_{0}^{r \pi t}\left(\delta n_{h}\right)^{2} r d r,
\end{aligned}
$$

invoking particle conservation and Eq. (4) above. The ratio of the neutron rates before and after the crash is:

$$
\frac{I_{b}}{I_{a}}=\frac{I_{b} r<r_{a t}+I_{r>r_{a l}}}{I_{a, r<r_{a t}}+I_{r>r_{a t}}}=1+\frac{k \int_{0}^{a \prime}\left(\delta n_{h}\right)^{2} r d r}{I_{a}} .
$$


Equation (5) implies that, if particles are conserved and velocities unchanged, the neutron emission must fall through ion mixing since $I_{b} / I_{a}>1$ for all $\delta n_{h} \neq 0$. In particular, since most of the fusion reactions occur in the sawtooth region,

$$
\frac{I_{b}}{I_{a}}-1 \lesssim \frac{k \int_{0}^{r a t}\left(\delta \pi_{h}\right)^{2} r d r}{I_{a, r<r_{u}}}=\frac{2}{r_{s t}^{2}} \int_{0}^{r_{u} \prime}\left(1-\frac{\pi_{h}(r)}{\bar{n}_{h}}\right)^{2} r d r .
$$

The density profile of the hot deuteriun is estimated assuming a temperature profile of the form $T_{d}(r)=T_{d 0}\left[1-(r / a)^{2}\right]^{\alpha_{T}}, A 1-d$ Maxtrellian velocity distribution has

$$
\begin{aligned}
\pi_{h}(r) & =\frac{n_{d}(r)}{\sqrt{\pi E_{h} T_{d}(r)}} \exp \left(-\frac{E_{h}}{T_{d}(r)}\right) d E \\
& \simeq r_{h 0} \exp \left(-\frac{\kappa r^{2}}{r_{t t}^{2}}\right), \text { where } \kappa=\frac{\alpha_{J} E_{h} r_{d t}^{2}}{T_{d 0} a^{2}},
\end{aligned}
$$

and $E_{h}$ is the energy of the deuterons responsible for most of the fusion emission [Eq. (3)]. After mixing, this distribution is flattened to a constant value $\bar{n}_{h}$, where, for particle conservation,

$$
\begin{aligned}
\tau r_{s e}^{2} \bar{n}_{h} & =2 \pi \int_{0}^{r \Delta x} n_{h}(r) r d r \\
\Rightarrow \bar{n}_{h} & \simeq \frac{n_{h 0}}{\kappa}\left(1-e^{-\kappa}\right) .
\end{aligned}
$$

With this hot-ion density profile, Eq. (6) gives

$$
\begin{aligned}
\frac{I_{b}}{I_{\alpha}}-1 & \leqslant \frac{2}{r_{\alpha}^{2}} \int_{0}^{r \cdot(}\left(1-\frac{\kappa e^{-\infty} / r_{1}}{1-\mathrm{e}^{-\kappa}}\right)^{2} r d r \\
& =\frac{\kappa}{2} \frac{\left(1-\mathrm{e}^{-2 \kappa}\right)}{\left(1-\mathrm{e}^{-\kappa}\right)^{2}}-1 \simeq \frac{\kappa^{2}}{12} .
\end{aligned}
$$

Thus the final expression for the magnitude of the drop in neutron emission due to ion mixing is:

$$
\frac{I_{b}}{I_{a}}-\mathrm{i} \lesssim \frac{1}{12} \alpha_{\tau}^{2}\left(\frac{E_{h}}{T_{d \infty}}\right)^{2}\left(\frac{T_{a t}}{a}\right)^{4}=O(10 \%)
$$




\section{Numerical Analysis}

The exact prediction of the mixing model for the magnitude of the fast drop in neutron emissicn at the sawtooth is evaluated numerically by performing the full six-dimensional velocity space integral [Eq. (2)] for the feactivity, using the mixed deuterium distribution. The distribution function is obtained by homogenizing the pre-crash spatial dependence of $f(r, v)$ in the mixing region:

$$
\begin{aligned}
& f_{0}(v)=\int_{r<r_{0,}} n_{d}(r) f_{M}(r, v) d V / \int_{r<r_{01}} n_{d}(r) d V \\
& =\frac{2}{\bar{n}_{d} r_{a k}^{2}}\left(\frac{m_{d}}{2 \pi}\right)^{3 / 2} \int_{0}^{r_{a z}} n_{d}(r) T_{d}^{-3 / 2}(r) \operatorname{erp}\left(-\frac{m_{d} v^{2}}{2 T_{d}(r)}\right) r d r
\end{aligned}
$$

where $\bar{n}_{d}$ is the pout-crash deuterium density in the mixing region from Eq. C3. The resulting distribution function, and its deviation from Marwellian, is shown in Fig. 11. The surplus at high and low velocities corresponds to excessea of hot and cold particles near $r=0$ and $r=r_{t}$, respectively. A shortage of particles at the new thermal velocity from the same region: leads to the deficit obeerved at $v / v_{\text {th }}=1$.

The pont-mixing neutran emission from the sawtookh region is then

$$
I_{a, r<r_{n}}=\frac{1}{2} \pi_{\alpha}^{2} V \frac{r_{\alpha}^{2}}{a^{2}} \iint f_{a}\left(v_{1}\right) f_{a}\left(v_{2}\right) \sigma(v) v d v_{1} d v_{2},
$$

where $V$ is the plarms volume. Comparison with the pre-awtooth neutron rate as determined from Eq. (1) (iritegrated in the sawtooth region with the Maxwellian reactivity) gives the drop resulting from ion mixing.

The decrease in zeutron rate predicted by this model is typically between half and two-thirds of the upper limit derived in the heuristic analysis [Eq. (9)], between $2 \%$ and $15 \%$ of the total neutron rate for typical discharge conditions.

\section{Comparieon with Experiment}

With a model of density flattening that includes simultaneous mixing, the drop predicted above would be expected to occur on the same time scale as the electron sswtooth, or $\sim 30 \mu \mathrm{sec}$ on PLT (Sec. IVA). In this scerivio, the detector signal would be limited by the frequency response of t'ie photomultiplier amplifier, but would still be three to four times faster 
than the observed rat (as evidenced by the fastest neutron fluctuations observed with this diagnostic system). Thus it is clear that the ion density flattening and velocity distribution mixing proceed at distinctly different rates. Empirically, it afpears that a drop of roughly the predicted mapnitude occurs on a longer, $0.5-1.0$ msec time scale. Comparing the magnitude of the drop at the end of :his phase with the predictions of the mixing madel, we find that the actua drop is comparable to that expected theoretically. For the sawtooth events shown in Fig. 1, discharge parameters have been entered in the calculation [Eqs. (10) and (11)] for comparison with the experimental data. The PLT case $\left(n_{\infty}=5.8 \times 10^{13} / \mathrm{cm}^{3}, Z_{e f f}=3.0\right.$, density shaping parameter $a_{n}=1.4$, sawtooth inversion radius $r_{0}=12 \mathrm{~cm}$, $T_{i 0}=3.2 \mathrm{keV}$, and ior temperature shaping parameter $a_{T}=3.0$ ) has an observed drop of $12.5 \%$ in the neutron rate, while the code predicts $12.0 \%$. The TFTR eare $\left(n_{n 0}=5.2 \times 10^{13} / \mathrm{cm}^{3}, z_{\text {eff }}=2.2, a_{n}=0.62, r_{0}=23\right.$ $\mathrm{cm}, T_{\text {io }}=2.5 \mathrm{keV}$, and $\alpha_{\mathrm{T}}=2.2$ ) has an observed drop of $8.3 \%$, with the code giving 4.4\%. The PLT inversion radius is inferred from ECE channels separated by $6 \mathrm{~cm}$ in major radius and thus has an accuracy of no better than one $\mathrm{cm}$ in either direction. As the ragritude of the fast drop in neutron rate is roughly proportional to the fourth power of the inversion radius [Eq. (6), along with the relation $r_{a} \simeq \sqrt{2} r_{\text {, }}$, which is derived in Appendix C], the precision of this estimate of $\delta I_{n} / I_{n}$ in the mixing phase is only about $\pm 30 \%$. The temperature profile exponent $\left(a_{x}=3\right)$ which gives the observed drop is convirtent with Doppler-broadening impurity-line meanurements ${ }^{21}$ of the ion temperature profile during ${ }^{3}$ He-minority ICRH, but the calculation is somerghat sensitive to mall variation in this parameter $\left[\alpha \alpha_{T}^{2}\right.$ in the heuristic andysis, Eq. (9)]. Despite the large uncertainty, the results do show that mixing of the deuterium distribution function gives a reduction in neutron emission of the order of that observed, and the data show that this reduction doen not always oecur concurseatly with the drop in electron temperature, implying that the mixing process for ions is $\sim 10$ times slower than for electrons.

It is passible that the mechanism responsible for ion miring involves parallel ion motion along the magnetic field subsequent to field-line reconnection. On PLT, $T$, begine increasing again soon after the sawtooth crash, suggesting that closed magnetic surfaces are reestablished on roughly a $30 \mu$ see time scale. Typical ion velocitie are sufficiently slow that it is difficult for the ions to mix uniformly this quickly. The observed duration of the mixing phase on PLI ( $500 \mu s e c$ ) corsesponds to $\sim 20$ toroidal transits 
of the ion fluid, or $\mathbf{5 0}$ transits of the fast fusion-producing deuterons.

On the other hand, the duration of the mixing phase on TFTR is similar to the duration on PLT at roughly equal temperatures (Table 1), despite the fact that a transit around TFTR takes twice as long as on PLT. This suggests that another aechanism may be dominant. Perhaps the rate of decay of the emission $i$. governed by diffusion of a fine-scale structure that exists immediately following the electron crash. Variations in this structure from sawtooth to sawtuoth might account for the rather large variations in decay rate observed on TFTR and DIII-D.

In conclusion, the magnitude of the initial trop in neutron emiscion is consistent with mixing of the ion within $\sim 0.5$ mee. The time scale for mixing may be determined by the parallel ion velocity, but it could be goversed by some other mechanism.

\section{Equilibration Phase}

After the density distribution is mixed, the ion theoretically approach a Marwellian distributon in an ion-ian equipartition time $\tau_{i i}{ }^{29}$ :

$$
\begin{aligned}
& \tau_{i i}=\frac{4.9 \mathrm{~ms}\left[T_{\mathrm{d}}(\mathrm{keV}) \mathrm{p}^{3 / 2}\right.}{Z^{*} T_{\mathrm{m}}\left(10^{13} \mathrm{~cm}^{-3}\right)}, \\
& \text { where } Z^{\bullet} \therefore \frac{1}{n_{e}} \sum_{f} n_{f} Z_{f}^{2}\left(\frac{2}{A_{f}}\right)\left(\frac{2 A_{f}}{2+A_{f}}\right)^{3 / 2} \frac{\ln \Lambda^{d / f}}{\ln \Lambda^{d / d}} \\
& \Rightarrow Z^{\bullet} \simeq \frac{4}{5}+\frac{1}{5} Z_{* / f} \text { for arygen impurity. }
\end{aligned}
$$

Bowever, the decay tirne of the neutran rate due to thermalization is not expected to coincide with $T_{i}$ since the ians that produce the fision resctions are generally moce energetic than typical ions. To entimate the actual rate, consider the distribution function created by uniform redistribution of lons within the miring region (Fig. 11). Describe this distribution by a Maxwellian bulk dissribution $f_{M}$ and a perturbed distribution $\delta f$. The neutron enission produced by this distribution consints of a steady rate $I_{n o}$ produced by the bulk Marwellian $\left(f_{u} \cdot f_{u}\right)$ and a decaying portion $\delta I_{n}$ associated with resctions between the perturbed distribution and the bulk $\left(\delta f \cdot f_{L}\right)$ and with itself $(\delta f \cdot \delta f)$. A numerical investigation shows that the dominant contribution to $\delta I_{n}$ is from particles near the peal of the perturbed distribution $\left(v \simeq 2.3 v_{t h}\right)$ and particles from the Maxwellian bulk 
distribution with $v \simeq 1.5 v_{\text {th }}$. The reactions are between particles moving in nearly opposite directions, with a relative velocity of about $4 v_{t h}$.

The fast portion of the perturbed distribution, with velocity $v_{h} \gtrsim 2.3 v_{t h}$ thermalizes more slowly than $\tau_{i i}^{-1}$ because of the $v^{3}$ dependence of the collision time. The hot ions lose energy at the slower rate $T_{h i}^{-1}$, where

$$
\pi_{h i}=\frac{1}{3} \sqrt{\frac{2}{\pi}} u_{h}^{3} T_{i i}\left\{\operatorname{erf}\left(u_{h}\right)-\frac{2}{u_{h}} \operatorname{erf}^{\prime}\left(u_{h}\right)\right\}^{-1} ; \quad\left(u_{h}=v_{h} / v_{c h}\right) .
$$

As $\eta_{\text {in }}$ represents the decay time-constant for the fusion-producing portion of the perturbed distribution, it is a reasonable approximation to the exponential decay time expscted for the neutrons [see Eqs. (15) and (16)]. For a total decay $\delta I_{n}$ due to velocity-space relaration,

$$
-\frac{\sigma I_{n}}{\left(d I_{n} / d t\right)} \simeq \eta_{h i} \simeq \frac{1}{3} \sqrt{\frac{2}{\pi}} u_{h}^{3} \tau_{i j}
$$

giving an expected decay time-constant of about $3 \tau_{i j}$.

\section{Numerical Amalyai of Equilibration}

A more quantitative numerical evaluation of the time evolution of the equilibration phase has also been performed to check the heuristic result of Eq. (14). In this study, the post-mixing distribution function $f_{0}(v)$ calerlated from Eq. (10) is allowed to evolve in time through collisional slowingdown and parallel diffision, according to the Folker-Planck equation. The full six-dimensional vel scity-space integral [Eq. (11)] for the neutron rate is calculated at specified time steps to model the actual time erolution of the neutron signal, asruming that the density profile remains flat.

The neutron rate die to the perturbed distribution funetion $\delta f$ interacting with a Maxwellian background is

$$
I_{n}=\frac{1}{2} \tilde{n} \frac{r_{i}^{2}}{a^{2}} \iint 2 f_{N}\left(v_{1}\right) \sigma f\left(v_{2}\right) \sigma v d^{3} v_{1} d^{3} v_{2}
$$

with the time derivative

$$
\frac{d I_{n}}{d t}=\bar{n}_{d}^{2} V \frac{r_{d t}^{2}}{a^{2}} \iint f_{w}\left(v_{1}\right) \frac{\partial \delta f\left(v_{2}\right)}{\partial t} \sigma v d^{3} v_{1} d^{3} v_{2}
$$

Here $\delta f=f_{a}-f_{w}$, where the Maxwellian is taken with the post-crash values $\bar{n}_{d}$ and $\vec{T}_{d}$ derived in See. IVB, conserving particles and energy in the sawtooth region. The evolution of the perturbed distribution function is given 
by the Folker-Planck collision operator. For an isotropic distribution encountering a Maxwellian background plasms with equal deuterium, electron, and impurity temperatures $T_{d}=T_{e}=T_{\text {imp, }}$

$$
\begin{aligned}
& \frac{\partial \delta f}{\partial t}=\sum_{f=e_{1} d_{j}, m_{\mathrm{p}}} \frac{m_{d}}{m_{f}} \nu_{0}^{d / f} u \frac{\partial}{\partial u}\left[\left(\delta f+\frac{1}{2 u} \frac{\partial \delta f}{\partial u}\right)\right. \\
& \times\left.\left\{\operatorname{erf}\left(\sqrt{\frac{m_{f}}{m_{d}}} u\right)-\text { uerf }\left(\sqrt{\frac{m_{f}}{m_{d}}} u\right)\right\}\right]
\end{aligned}
$$

where $\nu_{n}^{d / f}$ is a characteristic collision time

$$
\nu_{0}^{d / f}=\frac{4 \pi e_{d}^{2} e_{f}^{2} n_{f} \ln \Lambda^{d / f}}{m_{d}^{2} v_{d}^{3}},
$$

and $u$ again denotes $v / v_{\mathrm{ch}, d}$. The terms in the square bracket proportional to of and its derivative represent collisional slowing-down and parallel diffusion of the pertubed distribution, resectively, and obviously sum to zero for a Marwellian of.

The time evolution of the distribution function is shown in Fig. 12(a). The distribution decays in about one ion-ion equipartition time (3.5 $\mu$ ) for $v<1.5 v_{t h}$, but at higher velocities, $\left(v \gtrsim 2.3 v_{t h}\right)$, the decay time it conriderably longer. The result is that the neutroe rate $I_{n}$ decays quite slowiy; an upproximately exponertial decay with a churecteristic time-constant of 10.2 $m s$ is found for the cace shown, where $n_{n}(r)=5 \times 10^{15}\left(1-r^{2} / a^{2}\right)^{1.2} / \mathrm{cm}^{3}$, $T_{d}(r)=3\left(1-r^{2} / a^{2}\right)^{2.2} \mathrm{keV}, r_{t} / a=0.34$, and $Z_{\alpha / f}=2.5$. This is about 3 times $\tau_{\mathrm{fi}}$, consistent with the heuristic value given by Eq. (14). In addition, it is found that the expected drop for complete relaxation is only a few percent; typically between half and two-thirds of the expected drap from the mixing phase. The numerieal result for the $I_{n}$ time evolution is shown in Fig. 12(b), where Eq. (15) has been integrated at each time step for inte shot parameters ditcie. Tïe small contribution to tìve vieutrun emissiüs from the region $r>r_{*}$ in unchanged during the dimuption and las been omitted from the magritude of $I_{n}$ shown in Fig. 12(b). The total drop seen in Fig. 12(b) is only $1.7 \%$, compared with a drop in the mixing phase for these parameters of $2.5 \%$.

The time scale for velocity-space relacation is thus comparable to the full sawtooth period ( $\$ 20 \mathrm{nns}$ ) on PLT, and the small associated drop in neutron emission (relative to that during the mixing phase) implies that thermalization plays a minor rale in the observed time evolution of the PLT neutron 
sawtooth. In the time required for relaxation of the perturbed distribution, the centrally peaked density and temperature profiles are recovered, and auxiliary heating jegins to increase the neutron rate toward its presawtooth level. In TFTR ohmic discharges (Fig. 1(b)), Tit is shorter than in high-power ICRE conditions on PLT and the sawtooth period is much longer, so the equilibra:ion phase is allowed to reach completion before the heating phase takes over. In the relatively cold, dense H-mode discharges an

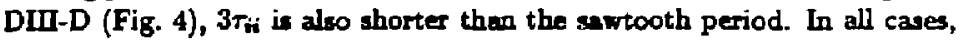
however, the predicted reduction in emission ascociated with thermalixation is relatively small and the predicted time scale of the reduction is relatively long, so it appears that other procrases such as ion transport (Sec. D) play a more important role in the evolution of the emjerion.

\section{Transport Phase}

Both the mropitude and the time evolution of the neutron emission suggest that after the initial rapid drop, tranport procesess becorse impartant. After the ion deasity ard temperature profiles bave flattened, a relatively large gradient in plarms density ard teinperature exists at the edge of the mixing region. The "shoulder" ditiuses away on a time scale determined by the local radial ion thermal conductivity and the size and slope of the discontimuity in ion temperature. In this model, while the steep gradient existn, the neutron rat, drope due to the lose of energy from the central region of the plarma, where esentially all of the fusion product geutrons are ban. In this rection, we ccompute the expected rate of change of the emission during this phase ior comparison with PLT data (Sec. 1) and compare the magritude of the drop with theoretical predictions (Sec. 2).

\section{Rate of Decay}

The $d(d, n)^{3} H e$ reactivity given by the Gamow cross section has a time derivative

$$
\frac{1}{\langle\sigma v\rangle} \frac{d(\sigma v)}{d t}=\left\{\frac{B}{3 T_{d}^{1 / 3}}-\frac{2}{3}\right\} \frac{1}{T_{d}} \frac{\partial T_{d}}{\partial t},
$$

with $B=19.87$ for $T_{d}$ in $\mathrm{keV}_{r}{ }^{27}$ implying a neutron decay rate due to thermal conduction of

$$
\tau_{n}^{-1}=-\frac{1}{\delta I_{n}} \frac{d I_{n}}{d t}=-\frac{1}{\delta I_{n}} \int \frac{1}{2} r_{d}^{2}\langle\sigma u\rangle \frac{1}{T_{d}} \frac{\partial T_{d}}{\partial t}\left\{\frac{B}{3 T_{d}^{1 / 3}}-\frac{2}{3}\right\} d V .
$$


Here $\delta I_{n}$ is the total drup in neutron rate during the transport phase. There is also a reduetion in emission associated with particle diffusion but, in this approximation, this term has been neglected.

At the onset of the transport phase, the temperature gradient is negligible everywhere except at the discontinuity radius, $r_{n}$. At this radius, the energy loss due to thermal conduction is

$$
\begin{aligned}
& 4 \pi^{2} R_{0} r_{\mu}\left[\frac{3}{2} n_{d} \frac{\partial T_{d}}{\partial t}\right] \Delta r=\int n_{d} x_{d} \frac{\partial T_{d}}{\partial r} \bar{r} \cdot d A=4 \pi^{2} R_{0} r_{s t} n_{d} X_{d} \frac{\partial T_{d}}{\partial r} \frac{\Delta r}{r_{s t}} \\
& \Rightarrow \frac{\partial r_{d}}{\partial_{t}}=\frac{2 x_{d}}{3 r_{d}} \frac{\partial T_{d}}{\partial r} \text {. }
\end{aligned}
$$

The surface integral is ove two toroidal shells of radii $r_{\Delta t}$ and $r_{\lambda t}+\Delta r$. Assuming that the coly contribution to the volume integral in Eq. (18) is from the discontimuous region,

$$
\tau_{n}^{-1}=-\frac{4 X_{d}}{3 r_{d t}^{2}} \frac{I_{n}}{\delta I_{n}}\left\{\frac{B}{3 T_{d}^{1 / 3}}-\frac{2}{3}\right\}\left[\frac{1}{T_{d}} \frac{\delta T_{d}}{\partial r} \Delta r\right]_{r_{.1}} .
$$

The term in square breckets in the change in the temperature profile accoss the discontinuity:

$$
\frac{1}{T_{d}} \frac{\partial T_{d}}{E_{r}} \Delta r \simeq-\frac{\alpha_{r}\left(r_{*}^{2}-r_{0}^{2}\right)}{a^{2}} \simeq-\frac{a_{r} r_{r t}^{2}}{2 a^{2}}
$$

for an ion temperature profile $T_{d}(r)=T_{d o}\left(1-r^{2} / \sigma^{2}\right)^{\infty r}$ flattened incide $r_{d}$ to the value $T_{d} \simeq T_{d o}\left(1-r_{0}^{2} / a^{2}\right)^{a r}$, and invaling Eq. (C5) for ro. Thus the expected time constant for decay of the neutron emission during this phase is

$$
\begin{aligned}
\tau_{n} & \simeq C_{1}\left(\frac{a^{2}}{\chi_{d} a_{s}}\right) \frac{\delta I_{n}}{I_{n}} \\
\text { where } C_{1} & =\frac{3}{2}\left\{\frac{B}{3 T_{d}^{1 / 3}}-\frac{2}{3}\right\}_{\text {r.t }}^{-1} \simeq 0.4 \text { for } T_{d} \simeq 3 \mathrm{keV} .
\end{aligned}
$$

The decay time is this characterized by the size of the sawtooth region $\left(r_{s t}^{2}\right)$, the steepness of the temperature gradient $\left(\alpha_{T} r_{k}^{2} / 2 a^{2}\right)$, and the local ion thermal conductivity $\chi_{d}$ at the saxtooth radius.

The peak-to-trougl. time $\tau_{p-t}$ of a sawtooth is dominsted by this trans. port time, since it is long compared to the duration of the mixing phase. 
Equation (19) suggests that $\tau_{p-s}$ should be expected to scale with the square of the minor radius of the device; a plot of observed values of $\tau_{p-c}$ for a number of machines is : hown in Fig. 7 . Assuming a typical ion temperature profile for these machires of $T_{d}(r)=2\left(1-r^{2} / a^{2}\right)^{2} \mathrm{keV}$, and $\delta I_{n} / I_{n}=0.1$, the coefficient of the $a^{2}$ fit implies an ion thermal diffusivity $x_{d}=7.5 \times 10^{3}$ $\mathrm{cm}^{2} / \mathrm{sec}$, which is reassnable. Equation 19 represents an estimate of the peak-to-trough time which is actually an upper bound on the expected decay time, since it peglects the effects of particle diffusion. In addition, $\tau_{p-t}$ carries a large experimental uncertainty, since the absolute minimum in the neutron emission may simply reflect the time at which the energy gain from awiliary heating overcomes the energy lows due to thermal conduction, rather than the end of the enhanced conduction phase.

A slight manipulation of Eq. (19) yields:

$$
-\frac{I_{n}}{d I_{n} / d t} \simeq \frac{3}{2}\left\{\frac{B}{3 T_{d}^{1 / 3}}-\frac{2}{3}\right\}^{-1} \frac{a^{2}}{x_{d} a_{\tau}}
$$

A plot of the right-haud side of this equation verous the observed neutron decay time-conatant in the transport phave is ahown in Fig. 13 for a series of ICRH dischargen on PIT, using Chang-Hinton neoclastical conductivity including impurity effests. ${ }^{30}$ The temperature-profile shaping-parameter is inferred from the initial reduction in neutron emistion, according to the preseriptions discussed below. The value of the neoclarsical conductivity is uncertain to $\pm 50 \%$ due to uncertainties in $Z_{\text {cff }}$ and $B_{f}$. Since the derivatian of Eq. (20) neglects partiele diffurion, the expected drop rate is actually 20\% larger (ansuming $D_{i} \sim X_{d}$ ). In addition, as the equilibration phase runs concurrently and at a similar rate, the actual drop rate is further enhanced $\sim 20 \%$. Thus, the duta in Fig. 13 are consistent with theory, indicating that thermal conduction probably is the dominant process governing the decay of the neutron emissica.

\section{Magnitude of Drop}

If it is assumed that no ion energy is lost from the mixing radius, the predicted reduction in neutron emiseion at a sowtooth is much smaller then observed. In this section, we compute the expected drop in neutron emisvion for various ion tempera ure profiles and show that the observed drop implies loss of ion energy frola the mixing region. Stringer has used a similar 
mixing model to analyze JET data With an implicit assumption of energy conservation, he too found that the drop in emission is larger than expected.

We begin by adopting a simple model that we subsequently show is inconsistent with the data. Assume that the ion density and temperature profiles within the mixing radius are flattened and that energy and particles are conserved. Experimentally, two quantities related to the ion temperature profile are measured: the neutron emission $I_{n}$ and the drop in emission at the sawtooth $\Delta I_{n}$. Since the deuterium density profile is known fairly accurately from electron density and $Z_{\text {off }}$ mearurements and, in any event, the neutron emission is lesn sensitive to denuity than to temperature, these two neutron meanurements would (if our anumptions were valid) allow us to infer two features of the ion temperature profile. We assume that the functional form of the ion temperature profilo is $T_{d}(r)=T_{d 0}\left(1-r^{2} / a^{2}\right)^{\alpha_{T}}$. Then the measurements of $I_{r}$,d $\Delta V_{n}$, together with the density measurements and measurement of the mixing radiu (infersed from ECE or soft $X$-ray measurements of the temperature inversion radius), permit unique determination of the central ion temperature $T_{\infty}$ and of the peakedness of the temperature profile $\alpha_{\mathbf{r}}$. Intuitively, for a fuxed level of neutron enission, if the drop in eminsion at the sawtooth in large, then the ion temperature profile must peak strongly on axit. Alternatively, if the initial profile is flat, a sowtooth renults in no reduction in emixion $\left(\Delta I_{n}=0\right)$.

Quantitative implications of the model are obtained numerically (Appendix C). In Fig. 14h, we plot the central ion temperaxure inferred from the drop in neutron eminion verrus the central ion temperature deduced by a code that usen the neutron emission and the assumption of a neoclassical ion diffurivity profile shape to infer $T_{\infty}$. The temperature inferred from the neutron drop is $\sim 25 \%$ langer than the values ce:-ulated by the analyzis code and also exceed the values mearured uring Doppler broadened impurity line radiation. For the neutron drop model, the mean temperature peaking p:rameter for this date set is $\left\langle\alpha_{r}\right\rangle=3.9$, which is much higher than predicted neoclarsically. Recently, the neoclaseical $\chi_{i}$ anumption has been shown to be violated on TFTR ${ }^{31}$ but not by an amount nearly this large. Even taking into account pouvible errors and uncertainties in other determinations of the ion temperature, the ion temperature profile inferred by our model is unrealistically peaked. This implies that some ion energy is lost from the miring region after the sawtooth.

The time evolution of the neutron emission of TFTR changes after 1 msec (Fig. 1b). At the time that the neutron emission changes slope, the 
emission has only fallet $\sim 1 / 3$ of the eventual drop. If we postulate that in this initial period the density and temperature profiles flatten, the computed ion temperature is consistent with the $T_{i}$ mearured by Doppler broadening (Fig. 14b). Using the asumption that only the initial drop is due to profile flattening, the average profile shaping parameter is $\left(\alpha_{\tau}\right)=2.2$. From the magnitude of the additional reduction in emission, the loss of energy from the mixing region is roughly $\Delta W_{d} / W_{d} \sim 3 \%$ for typical parameters. For PLT, using the full drop yields $\left\langle a_{\tau}\right\rangle=4.8$, while the analysis gives $\left\langle a_{T}\right\rangle=2.5$ if the initial neutron drop is used rather than the overall drop.

In conclusion, both the magnitude and the duration of the drop in $I_{n}$ suggest that energy is lost from the mixing region following the sawtooth.

\section{v. CONCLUSION}

At a sawtooth, the neutron emission typically falls $\sim 10 \%$ in $\sim 0.5 \mathrm{msec}$, indicating that the hot jons that produce thermcouclenr resctions generally take longer to mix thac electrons. After the initial rapid drop, the emission falls an additionel 10\% in 5-20 msec. Equilibration of the hot ions only accounte for one-fifth of this gradual drop. The data during this phase are consistent with the hypothesis that an ion heat pulse propagates away from the mixing region as the steep gradient in $T_{i}$ diffuses.

Empirically, the drop in neutron emiscion $\Delta I_{n} / I_{n}$ varies linearly with the drop in central electron temperature $\Delta T_{q} / T_{\varepsilon}$. Eatimates of the effect of sawteeth on the thermonuclear emisuion can be obtained from the scaling $\Delta T_{d} / T_{d} \simeq 0.4 \Delta T_{\varepsilon} / T_{e}$.

\section{ACKNOWLEDGMENTS}

The support of the PLT, TFTR, and DIII-D staff is gratefully acknowledged. Gary Estepp and John Evans fabricated the neutron detectors. Data were contributed by.M. Bitter, E. Fredrickeon, B. Grek, H. Hendel, D. Johnsnn, K. MeGuire, B. Park, A. Ramsey, and G. Taylor for TFTR; by R. Bell, A. Caiallo, P. Coleatoci, and R. Wilson for PLT; and by T. Carlstrom, J. Lohr, T. Osborne, and R. Snider for DIII-D.

This work ans supported by U.S. Department of Energy Contracts DEAC02-76-CHO-3073 acd DE-AC03-84ER51044. 


\section{Appendix A. Effect of Sawtooth on Beam-Plasma Neutron Emission}

To explain the time evolution of the thermonuclear neutron emission, we bave postulated several phases of ion response to the sawtoath event (Sec. IV). As a check on the validity of our model, we consider the expected effect of these phases on the neutron emistion during $D^{0} \rightarrow D^{+}$neutral beam injection, when the fusion reactions are beam-planms in nature. We find that the predictions are consintent with experimental observations.

During $D^{0} \rightarrow D^{+}$neutral beam injection, the neutron emiasion is dominated by beam-plasima reactions. Previous work on PLT ${ }^{\circ}$ found that the sawtooth instability causes a $\sim 3 \%$ drop in neutron emiscion in $\sim 5$ msec. On TFTR (Fig. A.1), the magitude of the drop is also only a few percent but the drop is $\sim 4$ times alower, varying between 10 and 45 mee. In PLT, the decay was sccounted for by the inereave in electron drag aweociated with flattening of the temperature profile." Flattening of the thermal decterium density also may have played a role." Nominally, the difference in decay time between TFTR and PLT (and DIII-D) in consistent with the increaced electron temperature and slowing-down time $\tau_{\text {, }}$ in TFTR. The decay time in TFTR does not appear to correlate with $\tau_{n}$, however.

As for thermonuclear neution sawteeth, the magnitude of the drop in enisvion $\Delta I_{n} / I_{n}$ seale linearly with the amplitude of the electron tem-

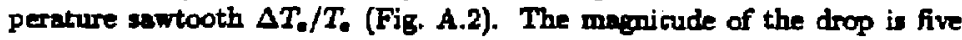
times waller than in the olmic case, however, scaling appromimately as $\Delta I_{n} / I_{n} \simeq 0.3\left(\Delta T_{e} / T_{0}\right)$.

For beam-plarma reactions, the neutron emistion is approximately

$$
I_{n} \simeq \int n_{b} n_{d} \overline{\sigma v}_{b d}
$$

where $n_{b}$ is the density of beam ions and $\overline{\sigma v}_{t-d}$ is the fusion reactivity for beam-plarma reactions. In steady-state, $\bar{\sigma}_{b d}$ depends principally on the beam injection energy $E_{b}$ and only weakly on the bulk deuterium tempereture $T_{d}$. The dependence of the reactivity on ion temperature can be estimated by evaluating the reactivity of a monoenergetic beam in a Maxwellian plasma, $\bar{\sigma} v_{b d}=\int f_{d}\left(v_{d}\right) d v_{d} \sigma v\left(\left|v_{b}-v_{d}\right|\right)$. The result is

$$
\bar{\nu}_{b d} \simeq \sigma v\left(E_{b}\right)\left[1+\left(\eta+\frac{1}{2}\right) \eta \frac{T_{d}}{E_{b}}\right],
$$


where $\sigma v\left(E_{b}\right)$ is the reactivity of the monoenergetic beam incident on a cold target and the cross sestion is assumed to vary approximately as ov $\propto E^{n}$ at $E=E_{b}$. At $E_{b}=80 \mathrm{keV}, \eta=2.2 \mathrm{so}$, for $T_{d} \simeq 2 \mathrm{keV}$, a $10 \%$ reduction in ion temperature only results in a $\$ 2 \%$ reduction in fusion reactivity.

If besm ions do not gain or lose enengy anomalously at a sawtooth, immediately following the sawtooth the fusion reactivity is virtually unchanged since the beam slowing-down time $\tau$, is long compared to the duration of the electron sawtooth. We assume that prior to the sawtooth the beam and deuterium densities are $\left.n_{b} ; r\right)=n_{b_{0}}\left(1-r^{2} / a^{2}\right)^{a_{b}}$ and $n_{d}(r)=n_{c_{0}}\left(1-r^{2} / a^{2}\right)^{a_{n}}$ and that the beam and deuterium densitied flatten of the electron sawtooth. Carrying out a derivation similar to that of Eq. (8), the expected reduction in beam-planma enistion associated with the flattening of the densities is

$$
\frac{I_{b}}{I_{a}}-1 \lesssim \frac{1}{12} a_{b} a_{n}\left(\frac{r_{a t}}{a}\right)^{4}
$$

Comparison with Eq. (8) indicates that the besm-plasma drop is smaller than the thermonuclear drop by a factor $a_{b} a_{n} / a_{z}^{2}\left(T_{d} / E_{h}\right)^{2} \simeq 0.1$. Thus, the expected reduction in benm-plann neutron emierion in the crash and mixing phave is $\leqslant 1 \%$. This is in good agreement with experiment, where esentially no change is observed on this time scale.

Following the sawtcoth crash, the beam distribution function chenges in response to changes in Coulomb drag and the reactivity and besm density become function of time. The time aceie for this trancitional period is governed by the alowing-down time $T_{r}$. This time scale is several times longer than the equilibration time for hot thermal ions. Since $r_{\text {, depends }}$ on electron temperature, the flattering of $T_{\text {s }}$ tends to reduce the neutron emissivity within the inversion radius and inereave it outside the mixing radius. The beam density is peaked on axis so a net reduetion in emission is expected but the volume-averaged nature of the neutron measurement reduces the predicted reduction to only a fraction of $\Delta T_{e} / T_{\mathrm{e}}{ }^{\circ}$ Because of the walk dependence of the reactivity on ion temperature [Eq. (A2)], the reduction in $T_{d}$ associated with the transport phase is expected to have a very small effect on the neutron source strength. Experimentally, the drop in emission does take place on the time scale of $\tau_{0}$ but the drep time does not correlate with variations in $r_{5}$, perhaps indicating that changes in beam and ion density profiles occur on a similar time scale. The drop in emiasion is typically $30 \%$ of the "eduction in electron temperature, in good agreement with theory. 
Appendix B. Effect of Sawtooth on $15 \mathrm{MeV}$ Proton Em:s sion

During ${ }^{3} \mathrm{He}$-minority ICRH heating the ${ }^{3} \mathrm{He}$ ions are heated to high energies and produce $15 \mathrm{MeV}$ protons in beam-plasma reactions with the thermal deuterium ions. Previous measurements ${ }^{22}$ of the volume-integrated proton rate made with a bottom-mounted, collimated surface-barrier detector on PLT showed little or no sawtooth activity, while a side-mounted proton probe did see reductions in $\mathrm{d}^{3} \mathrm{He}$ proton emission correlated with drops in central soft $x$-ray and neutron emission at sawtooth crashes. The interpretation of the difference between the retector signals was that the de-

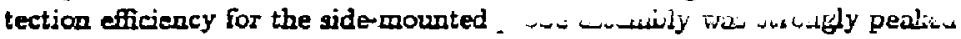
near the plasma center. while the bottom-mounted detector integrated over about half of the piams minor radius, and therefore over the entire sawtooth region ${ }^{22}$ A proton detector on $\mathrm{JET}^{23}$ with a detection efficiency that is peaked outside of the $\mathrm{d}\left({ }^{3} \mathrm{He}, \mathrm{p}\right) \alpha$ source region has also shown bursts in signal level at sawtcoth crashes, suggesting that energetic ions are expelled from the central region by the sswtocth.

New measurements of the $15 \mathrm{MeV}$ proton emiswion were made with a detector mounted in the bottom of the PLT vacuum vessel in a geometry similar to that employed previously. 20 These measurements give direct evidence for deraterium dencity flattening on the time scale of the eleciron temperature sawtooth. The experimental condition for these recent mearurements are $P_{\text {RF }}=2.5 \mathrm{MW},{ }^{3} \mathrm{He}$ ninority concentration $\simeq 5 \%, \bar{n}_{\mathrm{w}}=3.7 \times 10^{13} \mathrm{~cm}^{-3}$, and $T_{0}=1.5 \mathrm{keV}$. Estimate based on Stix's theory 32 suggest that these conditions result in a perpendicular ${ }^{3} \mathrm{He}$ tail temperature of $\sim 130 \mathrm{keV}$. Weighting by the fant-rising fusion cros section, the average energy of the ${ }^{3} \mathrm{He}$ ions responsible for fusion emission is $\sim 400 \mathrm{keV}$ for these parameters. Theory ${ }^{32}$ and experiment ${ }^{35}$ indicate that the ${ }^{3} \mathrm{He}$ ions are eccelerated in perpendinular velocity until their orbits becone trapped, and then further accelerated until the benana tips migrate outward in majos radius to the location of the resonance layer $\boldsymbol{R}_{\text {res }}$ (Fig. B.1). Particles on these orbits spend most of their time at the turning paints, causing a so-called "resonancelocalization" of the minority, and implying that most of the $\mathrm{d}-{ }^{3} \mathrm{He}$ fusion occurs at or near the resonance layer. Experimental meanurements of the $\mathrm{d}^{3} \mathrm{He}$ spatial distribution ${ }^{34}$ and anisotropy ${ }^{35}$ are consistent with this prediction. As the $400 \mathrm{keV}{ }^{3} \mathrm{He}$-ion gyrodiameter is approximately $5.4 \mathrm{~cm}$ at resonance ( $B_{t}=29.46 \mathrm{kG}$ for a $30 \mathrm{MHz}{ }^{3} \mathrm{He}$-ion cyclotron resonance), the 
d- ${ }^{3} \mathrm{He}$ proton source region is assumed to be the $\sim 5 \mathrm{~cm}$ of major radius outside of $R_{\text {res. }}$. Depenting on the relative position of the ICRH resonance layer and sawtooth inversion radius, the emission is observed to decay slowly (Fig. B.2a), increase gradually (Fig. B.2b), or increase rapidly (Fig. B.3). In Fig. B.3, the relative jumps are $5 \%$ in $30 \mu \mathrm{sec}, 4 \%$ in $30 \mu \mathrm{sec}$, and $4 \%$ in $\because 0 \mu \mathrm{sec}$ for the three events shown. These results are evidence for flattening of the deuterium density at a sawtooth.

The effect of density flattening on the $\mathrm{d}^{-3} \mathrm{He}$ rate is determined in a manner similar to that discussed for the $d-d$ rate in See. IVB. The denterium density is multiplied by the reactivity-weighted ${ }^{3} \mathrm{He}$-ion density and integrated over the source region before and after a sawtooth. Prior to the sawtooth, the electron temperature profile is assumed to be $T_{e}(r)=\left(1-r^{2} / a^{2}\right)^{2}$ and the electron, deuterium, and ${ }^{3} \mathrm{He}$ densities are taken to be parabolic, implying a reactivity-weighted ${ }^{3} \mathrm{He}$-ion density which is roughly parabolic to the second power in the source region, and small outside. The actial profile may be steeper, as arial focussing of the RF wave power by the antennae has been neglected. After the sawtooth erash, the deuterium density profile is assumed flattened or the time scale of the electron sawtooth. Depending on the position of the resonance-layer with respect to the sawtooth inversion radius, the relative fractions of the source region in which the target density is increased and decreased (the hatched regions in Fig. B.1) are determined. Thus it is possible for she proton rate to decline, remain constant, or even increace at the crach, but the magnitude of this change is never more than a few percent (Fig. B.4). The change should occur on the time scale of the ion density flattening, or about $30 \mu$ (not the slower mixing time responsible for the neutron sawtooth crash, since the deuterium is a cold target relative to the energetic ${ }^{3} \mathrm{He}$ ). This prediction is consistent with experimental observations (Fig. B.3).

The proton sawtooth behavior subsequent to the electron sawtooth is romplex. The emission may change due to changes in the energy loss time $\tau_{d}$, which alters the balance between RF acceleration and collisional deceleration of the tail ions. As the electron density and temperature profiles are flattened at a sawtooth, $\tau_{\text {a decreeses for }} r<\tau_{0}$, thereby decreasing the equilibrium energy of the ${ }^{3} \mathrm{He}$ ions in this region. The opposite is true in the region $r_{0}<$ $r<r_{*}$, where both $r_{e}$ and the ${ }^{3} \mathrm{He}$ tail temperature increase. Depending on the relative portion of the source volume which falls into these two regions, the net proton emission could be expected to rise or fall. The bottommounted PLT detector which integrates over the full extent of the sawtooth 
and resonance layer, observes both behsviors at sowtooth cventa. The proton signal shown in Fig. B.2(b) increases about $10 \%$ at the neutron sawtooth, suggesting an average increase of about $6 \%$ in energy loss time $\tau_{e}$ which is consistent with the assumed profile shapes given an inversion radius a few cm cutside of the resonance layer at the plama midplane. The toroidal field is again $32.6 \mathrm{kG}$ for this discharge, but the sawtooth region is smaller than that for the discharge shown in Fig. B.3, which may explain the difference in time evolution.

Appendix C. Ion Temperature Profle Inferred using a Simple Mixing Model

Asnume that the ion temperature and density profiles are of functional form $T_{d}(r)=T_{d b}\left(1-r^{2} / a^{2}\right)^{\alpha r}$ and $n_{d}(r)=r_{\infty}\left(1-r^{2} / a^{2}\right)^{a_{n}}$ respectively, that the profiles are flattened at a sawtooth, and that energy and particles are conserved. Then measurements of neutron enission $I_{n}$ and reduction in emisaion $\Delta I_{n}$, together with measurements of the density profile and mixing radius suffice to determine the central ion temperature $T_{\text {ob }}$ and profile shape $a_{\mathbf{T}}$.

Assuming a Gamon form for the fuxion cross section, ${ }^{27}$ the neutron emission $I_{n}$ is

$$
I_{n}=\frac{1}{2} A \int n_{d}^{2} T_{d}^{-2 / 3} \exp \left(-B T_{d}^{-1 / 3}\right) d V,
$$

where, for the $\mathrm{d}(\mathrm{d}, \mathrm{n})^{3} \mathrm{He}$ reaction, $A=2.93 \times 10^{-14} \mathrm{keV}^{2 / 3}$ and $B=$ $19.87 \mathrm{keV}^{1 / 3}$. Substitution of the assumed radial profiles for $T_{d}$ and $n_{d}$ yields

$$
I_{n}=V n_{d 0}^{3} A T_{d 0}^{-2 / 2} \int_{0}^{1} x\left(1-x^{2}\right)^{C} \exp \left[-B T_{\infty 0}^{-1 / 3}\left(1-x^{2}\right)^{-D}\right] d x,
$$

where $x=r / a, C=2 a_{n}-\left(2 \alpha_{T} / 3\right)$, and $D=a_{T} / 3$. The integral is treated asymptotically using the Laplace method, expanding the integrand around $x=0$, where the exponential has a maximum:

$$
\begin{aligned}
\int & \sim \int_{0}^{a} z\left(1-C x^{2}+\cdots\right) \exp \left[-B T_{d 0}^{-1 / 3}\left(1+D x^{2}+\cdots\right)\right] d x \\
& \sim \mathrm{e}^{-B T_{\infty}^{1 / 3}} \int_{0}^{\infty}\left(2-C x^{3}+\cdots\right)\left(1-\frac{D(D+1)}{2} x^{4}+\cdots\right) \mathrm{e}^{-D B T_{\infty}^{-1 / 3} w^{2}} d x .
\end{aligned}
$$


Carrying out the integration to first order in the small parameter $T_{d 0}^{1 / 3} / B$, the result is obtained:

$$
I_{n} \sim \frac{S_{n 0} V}{1+2 a_{n}+\left(\frac{B T_{0}^{-1 / 3}-1}{3}\right) a_{T}},
$$

where $S_{n 0}=(1 / 2) n_{d 0}^{2} A T_{d 0}^{-2 / 3} \exp \left(-B T_{d 0}^{-1 / 3}\right)$ is the central neutron rate per unit volume. Equation (C2) is particularly illuminating in that it shows the relative effects of the temperature and density profile shapes on the neutron rate. For a $3 \mathrm{keV}$ plarms the temperature absing parameter $a_{T}$ in the denominator is multiplied by 4.25 , so the central temperature inferred from the neutron emission is twice an sensitive to temperature-profile shape than to density-profile shape. Although the error in the aymptotic formuls is in all cases lea than $1.5 \%$ of the exact wlution from numerical integration of Eq. (C1), we use the ecact solution.

A second relation, required to obtain a unique solution for the twoparameter ion temperature profile, is afforded by the magnitude of the drop in neutron emiscion. By integrating Eq. (C1) numerically in the mixing region to determine the pre-crah neutron rate, and evaluating the post-mixing neutron rate according to a preseribed model, the drop pre-

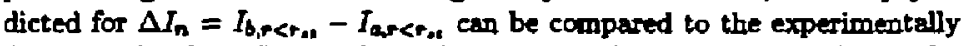
determined value. In matehing the observed date, a unque colution for $T_{d}(r)=T_{d}\left(1-r^{2} / a^{2}, a_{r}\right.$ is asfured, since for any particular value of $T_{d}$, $\delta I_{n}$ increasea with increasing $a_{r}$, while $I_{n}$ decreases. Thu the two curves describing the $\left(\alpha_{r}, T_{\infty}\right)$ solution space for the observed neutron rate and its reduction at the sawtooth crose at a single point (Fig. C.1).

The deuterium density profile flattens during the mixing phase to the constant value $\bar{n}_{d}$ which conserves particles inside the mixing radius:

$$
\begin{aligned}
\pi r_{d !}^{2} \bar{n}_{d} & =\int_{0}^{n_{n}} \pi_{d}(r) d^{2} r \\
\Rightarrow \bar{n}_{d} & =\frac{n_{d \infty}\left[1-\left(1-z_{\pi t}^{2}\right)^{\alpha_{n}+1}\right]}{x_{t t}^{2}\left(\alpha_{n}+1\right)} .
\end{aligned}
$$

The post-crash "equivaient temperature" profile is also flattened (the mixed distribution is non-Mai.wellian), to a value $\bar{T}_{d}$ which conserves energy inside the mixing radius:

$$
\pi r_{d t}^{2} \bar{n}_{d} \bar{T}_{d}=\int_{0}^{r_{d t}} n_{d}(r) T_{d}(r) d^{2} r
$$




$$
\Rightarrow \bar{T}_{d}=\frac{T_{d 0}\left[1-\left(1-x_{t t}^{2}\right)^{\alpha_{n}+\alpha_{T}+1}\right]\left(\alpha_{n}+1\right)}{\left[1-\left(1-x_{x t}^{2}\right)^{\alpha_{n}+1}\right]\left(\alpha_{n}+\alpha_{T}+1\right)} .
$$

Here $z_{\Delta t}=r_{a t} / a$. The sawtcoth radius $r_{s t}$ is not an experimentally measured quantity; instead the sawtooth size is characterized by the so-called "inversion radiu," $r_{0}$, at which the response of the soft x-ray emission (corrected for lineaveraging) and ECE emission to the sawtooth instability changes sign. Inside of $r_{0}$, the electron and ion densities and temperatures drop, accompanied by a drop in $x$-ray and electron cyclotron emission. Becween $r_{0}$ and $r_{n t}$ the densities and temperatures rise, and the I-ray and electroncyclotron emissions increase accordingly. The densities remain constant at the inveraion radiun, wo that by definition $n_{d}=n_{d 0}\left(1-r / a^{2}\right)^{\alpha_{m}}$. A comparison with Eq. (C3) gives, to first order in $r_{0} / a$ :

$$
\begin{aligned}
r_{s t}^{2} & =2 r_{0}^{2}\left(1-\frac{\left(a_{n}-1\right)}{6} \frac{r_{0}^{2}}{a^{2}}\right) \\
\Rightarrow r_{s t} & \simeq \sqrt{2} r_{0} .
\end{aligned}
$$

The relative error introduced by the last approximation is leat than $1 \%$, since typically $\alpha_{n} \sim 1$ and $r_{0}<a / 3$.

Assuming a Maxwellian distribution exiats immediately after the mixing phase, the neutron rate in the mixing region is

$$
I_{0, r<, t}=S_{n} V x_{\alpha}^{2} \text {, }
$$

where $\bar{S}_{n}=(1 / 2) \bar{n}_{d}^{2} A \bar{T}_{d}^{-2 / 3} \exp \left(-B \bar{T}_{d}^{-1 / 3}\right)$ is the constant source rate per unit volume throughout the region. 


\section{References}

1J. Schmidt, G. Batercan, C. Bushnell et al., in Proceedings of the Eleventh International Conference on Plasma Physics and Controlled Nuclear Fusion Research (IAEA, Kyoto, 1986), Vol. III, p.259.

${ }^{2}$ A. Gondhalekar et al., in Proceedings of the Seventh International Conference on Plasma Physics and Controlled Nuclear Fusion Research (LAEA, Innsbruck, 1978), Vol. I, p.199; D. Gwinn and R. Granetz, MIT Report RR-78-8 (1978).

${ }^{3}$ Equipe TFR, in Proceedings of the Ejghth International Conference on Plasms Physics and Controlled Nuclear Fusion Research (LAEA, Brussels, 1980), Vol. II, p.547.

'T. E. Stringer, Neutron Yield During Sawtooth Oscillations, JET Joint Undertaking Report No. JET-IR(86)04, (1986).

5J.M. Adarns, O.N. Jarvis, J. Kallne et al, Proc. Fourteenth Eur. Conf. on Cont. Furion Plarma Phys. (Madrid, 1987), Vol. II, part 3, p. 1224.

"M Murakami et a'., Plasmn Phys. Cont. Fusion 28, 17 (1986).

${ }^{7}$ G. Gammel, R. Kaita, R. Fonck, K. Jaehnig, and E. Powell, Rev. Sci. Instrum. 57, 1800 (1986).

'F. H. Seguin, R. Petranto, and E. S. Marmar, Phys. Rev. Lett., 51, 455 (1983).

'J.D. Strachan et al., Nucl. Fusion 21, 67 (1981).

${ }^{10}$ W.W. Heidbrink, Rev. Sci. Instrum. 57, 1769 (1986).

${ }^{11}$ J.D. Strachan et al., Nucl. Fusion 25, 863 (1985).

${ }^{12}$ W.W. Heidbrink et al., Phys. Rev. Lett. 57, 835 (1986).

${ }^{13}$ R. Kaita et al, Nucl. Fusion 26, 863 (1986).

${ }^{14} \mathrm{~K}$. McGuire et al., Froc. 12th European Conf. on Controlled Fusion and Plasma Physics (Budapest, Hungary) Contr. paper I, 134.

${ }^{15} \mathrm{~K}$. Burrell et af., Phys. Rev. Let:. 59, 1432 (1987). 
16J.D. Strachan, Proc. of the Course on Diag. for Fusion Reactor Conditions, Varenns (1982).

${ }^{17}$ W. Stodiek, R. Goldston, N. Sauthoff et 1., in Proceedings of the Eight.j International Conference on Plarms Physics and Controlled Nuclear Fusion Research (LAEA, Brussels, 1980), Vol. I, p.9.

18. J. D. Strachan et al., Nature 279, 626 (1979).

${ }^{20} \mathrm{~J}$. Hosea et al, in Proceedings of the Eighth International Conference on Plasma Physics and Controlled Nuclear Fusion Research (LAEA, Brussels, 1980), Vol. II, p.95.

${ }^{20}$ T. Nishitani, J.D. Strachan, Neutron Spectroncopy on TFTR, Princeton Plasma Phys. Lab. Rep. PPPL-2512 (1988).

${ }^{21}$ S.K. Kim, D.L. Brower, W.A. Peebles, N.C. Luhmann, Jr., Phys. Rev. Lett. 60, 577 (1988).

${ }^{22}$ R. E. Chrien and J. D. Strachan, Phys. Fluids 26, 1953 (1983).

${ }^{23}$ G. Martin, O. N. Jarvis, J. Källne, V. Merlo, G. Sadler, and P. van Belle, Physica Scripta T16, 171 (1987).

${ }^{24}$ R. J. Goldston, R. Kaita, P. Beiersdorfer et al., Nucl. Fusion 27, 921 (1987).

${ }^{25}$ E. L. Bydder, B. S. Liley, and A. H. Marton, Plasma Phys. 21, 669 (1979).

${ }^{20}$ H. Brysk, Plasma Phys. 15, 611 (1973).

${ }^{27}$ G. H. Miley, H. H. Towner, and N. Ivich, Fusion Cross Sections and Reactivities, University of Illinois Repart No. COO-2218-17 (1974).

${ }^{28}$ P. L. Colestock, IEEE Trans, Plasms Sci. PS-12, 64 (1984).

${ }^{29} \mathrm{~L}$. Spitzer, Jr., Physics of Fully Ionized Gases, (Interscience, New York, 1962), p. 135.

${ }^{30}$ C. S. Chang and F. L. Finton, Phys. Fluids 29, 3314 (1986).

${ }^{31}$ R.J. Foncl, S. Scott, M. Bitter et al., Ion Thermal Confinement in the TFTR Enhanced Confinement Regime, to be published (1988). 
${ }^{32}$ T.H. Stix, Nucl. Fusion 15, 737 (1975).

${ }^{33}$ R. Kaita et al., Nucl. Furion 23, 1089 (1983).

${ }^{34}$ T.J. Murphy and J.D. Strachan, Nucl. Fusion 25, 383 (1985).

${ }^{35}$ W. W. Heidbrink, Nucl. Fusion 24, 636 (1984).

${ }^{38}$ H.H. Towner, R.J. Goldston, Bull. Am. Phys. Soc. 29, 1305 (1984). 
TABLE 1. Thermonuclear Neutron Sawtooth Behavior

\begin{tabular}{|c|c|c|c|c|c|c|c|c|}
\hline \multirow[b]{2}{*}{ Tolumin } & \multirow[b]{2}{*}{ a(cm) } & \multirow[b]{2}{*}{$\boldsymbol{n}(\mathrm{cm})$} & \multirow{2}{*}{$\begin{array}{l}\text { Electron } \\
\text { Crash(ns) }\end{array}$} & \multicolumn{2}{|c|}{ Bapid Drop } & \multicolumn{2}{|c|}{ Total Drop } & \multirow{2}{*}{$\begin{array}{l}\text { Sawtooth } \\
\text { Period(nis) }\end{array}$} \\
\hline & & & & Bele(mu) & Megnitude" & Durution $(m s)^{c}$ & Megniturted & \\
\hline PLT & 10 & 132 & $\sim 0.03$ & $0.3-0.7$ & $\approx 60 \%$ & $\sim 5$ & $\sim 15 \%$ & $\sim 15$ \\
\hline DJJJ-D & 67 & 167 & $\sim 0.20$ & $0.3-1.5$ & $\sim 20 \%$ & $\sim 7$ & $\sim 32 \%$ & $\sim 30$ \\
\hline TFTR & 80 & 266 & $\sim 0.30$ & $0.3-1.2$ & $\sim 90 \%$ & $\sim 20$ & $\sim 13 \%$ & $\sim 130$ \\
\hline
\end{tabular}

$\Delta I_{n} /\left(d I_{n} / d t\right)$ during tapid drop in emivion

- Frection of colel drop $\Delta I_{n}$

$t_{p-t}$

${ }^{d} \Delta I_{n} / I_{n}$ for $\Delta T_{n} / T_{n}=20 \%$ 


\section{Figure Captions}

Fig. 1 Sawtooth in the neutron emission on (a) a PLT discharge with $3.2 \mathrm{MW}$ ICRH heating in a "He-minority plasma with $B_{\mathrm{t}}=33.2 \mathrm{kG}, I_{p}=$ $580 \mathrm{kA}, T_{\infty 0}=2.7 \mathrm{keV}, \bar{r}_{1}=3.6 \times 10^{13} \mathrm{~cm}^{-3}$, and $Z_{e f f}=3.0$ (ICRH shutoff at $630 \mathrm{~ms}$ ); and (b) a TFTR ohmic discharge with $B_{t}=47.1 \mathrm{kG}, I_{p}=2.2$ MA, $T_{\infty}=2.9 \mathrm{keV}, \bar{r}_{-}=3.9 \times 10^{1.9} \mathrm{~cm}^{-3}$, and $Z_{a f f}=2.2$. The initial drop rates and magnitudes are comparable, while the peak-to-trough time is approximately 4 times longer on TFTR, and the TFTR sawtooth period ( $130 \mathrm{~ms}$ ) is about 8 times longer.

Fig. 2 Neutron sawtooth and concurrent electron temperature sawtooth for a $3.2 \mathrm{MW}$ ICRH discharge on PLT with $B_{t}=33.2 \mathrm{kG}, I_{p}=580 \mathrm{kA}$, $\bar{n}_{1}=3.6 \times 10^{13} / \mathrm{cm}^{3}$, and a $3 \%{ }^{3} \mathrm{H}_{\mathrm{e}}$ minority. The average centrel electron temperature is $T_{\infty 0}=2.6 \mathrm{keV}$, while the average central ion temperature inferred from the neutron emission is $T_{d \mathrm{~d}}=3.2 \mathrm{keV}$. The electron temperature drop time is about $30, u s$, compared with an initial drop time of about 550 ss for the neutrons.

Fig. 3 Time derivative of the neutron rate shown in Fig. 1(a), rhowing a discontimuity between 450 and $850 \mu$ after the beginning of the sswtooth. The decay rate prior to this time (mixing phase) is $\sim 5 \mathrm{~ms}$, compared with $\sim 34 \mathrm{~ms}$ afterward (equilibration and transport phases). The points shown are the numerical derivative of a spline fit to $n$ ten-point-average of the 100 kFa dasa. The amplifier response is about an order of magaitude faster than the fast drop rate.

Fig. 4 Neutron sawtooth during $B^{0} \rightarrow D^{+}$beam injection in an $\mathrm{H}-$ mode plarms in DII-D. $I_{p}=1.25 \mathrm{MA} ; B_{\mathrm{t}}=2.1 \mathrm{~T} ; \bar{n}_{\mathrm{z}}=8.0 \times 10^{25} \mathrm{~cm}^{-3}$; $P_{\mathrm{b}} \simeq 7 \mathrm{MW}$.

Fig. 5 Central soft x-ray and neutron signals during the sawtooth shown in Fig. 4.

Fig. 6 Peak-to-trough decay time versus sawtooth period for PLT plasmas with various types of heating. The drop in neutzon emission, $\Delta I_{n} / I_{n s}$ also scales with the sawtooth period.

Fig. 7 Peak-to-trough decay time $t_{p-t}$ vs. machine minor radius $a$ on Alcator $A,{ }^{2}$ TFR, ${ }^{3}$ PLT, DIII-D, TFTR, and JET. The line is an $a^{2}$ scaling, suggenting a dependence on radial transport. For Alcator $A$, the meanurement of Tp-e may have been affected by the instrumental resolution. The error bars for TFR, PLT, DIII-D, and TFTR indicate the range of values observed; for JET, the error bar indicates the variation in a single discharge. 
In non-eircular plasmas, the short axis is used.

Fig. 8 Fractional drop in neutron emission versus fractional drop in electron temperature for obmic TFTR discharges. The points are averages over many sowteeth.

Fig. 9 Four phaces of neutron antooth crash: (a) crash phese, in which the ion density profile flattens to preserve quarineutrality; (b) mixing phase, in. which the ion distribution is homogenized in the sawtooth region; (c) equilibratior phase, in which a Maxwellian velocity distribution is reestabliahed; and (d) thermal transport phase, in which the "shoulder" on the ion temperature profile diffues away.

Fig. 10 Local redistribution of deuterons without significant mixing, implying little change in fusion neutron eminion. Each shaded region moves outward by one radial shell (to the corresponding shaded region) at the sawtooth. At the inversion radius ( $r=10 \mathrm{~cm}$ in the diagram), an integrated density corresponding to the entire excess from the central portion of the pre-crash profile is displaced by one radial step. The minimum radial step size consistent with local density redintribution is thus given by the interval $\Delta r$ for which a shell at the inversion radius contains a total mumber of icons equal to this ion excess. For a parabolic density profile and an inversion radius at $a / 4, \Delta r=a / 240$, or $0.17 \mathrm{~cm}$ on PLT. A flattening of the ian density occurring through radial displscements of this size should show little (< 1\%) change in neut:on emixion.

Fig. 11 (a) Pont-mixing deuterium dietribution function given by Eq. (10) for a planuas with $n_{d}=3 \times 10^{13}\left(1-r^{2} / a^{2}\right)^{1.2} \mathrm{~cm}-3, T_{d}=3\left(1-r^{2} / a^{2}\right)^{2.3}$ $\mathrm{keV}$, and $r_{\star} / a=0.25$, along with a Maxwellian with the same total energy [the Maxwellian is given by $\pi^{-1 / 2} \exp \left(-u_{2}^{2} T_{d 0} / T_{d}\right)$, where $\vec{T}_{d}$ is the flat-profile temperature given by Eq. (C4)]. (b) Difference distribution between the perturbed distribution and Marwellian, with flat-profile density given by Eq. (C3). The integral under this curve is zero, as is required for particle conservation.

Fig. 12 (a) Time evolution of the perturbed distribution function of Fig. 11, using the Folder-Planclk collision operator. The distribution decays in about $\tau_{i i}(3.5 \mathrm{~m})$ for values of $u=v / v_{t h}<1.5$, but the hot fusionproducing portion decays much more slowly, in about $3 r_{\mathrm{ii}}$. (b) Corresponding time evolution of the neutron rate, as determined from Eq. (15). The calculated drop is approrimately exponential, with a characteristic decay time of 10.2 ms and a relative magnitude of $1.7 \%$.

Fig. 13 RHS of Eq. (20) versus the observed neutron emission decay 
time-constant $-I_{n} /\left(d I_{r} / d t\right)$ during the sawtooth transport phase for a series of ICRH discharges on PIT using Chang-Hinton neoclassical conductivity including impurity effects. ${ }^{30}$ The line indicates equality between calculated and experimental drop times. Equation (20) neglects the effects of particle diffusion and velocity-space equilibration.

Fig. 14 (a) Cent:al ion temperature inferred from the drop in neutron emission versus the central ion temperature calculated by the SNAP ${ }^{36}$ analysis code in ohmic TFTR discharges. The neutron drop temperature is only valid if energy and particlea are converved. The SNAP calculation asnume that the shape of the ion temperature profile is determined by neoclansical diffurivity multiplied by a "neoclassical muitiplier." (b) Central ion temperature inferred ausuming only $1 / 3$ of the neutron drop is due to profile flattening versus SNAP temperature.

Fig. A.1 Sawtooth oscillations in the neutron emission during $D^{\circ} \rightarrow D^{+}$ neutral beam injection into TFTR from 2.5 to 3.0 seconds. $I_{p}=1.8 \mathrm{MA}$; $B_{\mathrm{t}}=4.8 \mathrm{~T} ; \bar{\pi}_{\mathrm{a}}=3.0 \times 10^{13} \mathrm{~cm}^{-3} ; T_{\mathrm{a}}(0)=4.4 \mathrm{keV} ; \Delta T_{\mathrm{a}} / T_{\mathrm{a}}=12 \% ; P_{\mathrm{b}}=$ $4.3 \mathrm{MW} ; E_{b} \simeq 76 \mathrm{keV}$; co-injection. The magnitude of the total drop is only $3 \%$.

Fig. A.2 Fracticnel change in neutron emission versu fractional change in central electron temperature during $D^{0} \rightarrow D^{+}$neutral beam injection into TFTR. The points are sverages over many sawteeth.

Fig. B.1 Guiding-center crbits of $200 \mathrm{keV}$ ions tangent to the resonancelayer for 30 MRz RF vaves in a 32 \&G PLT discharge. The shaded ares is the source region for the majority of the $\mathrm{d}(\mathrm{H}, \mathrm{P}, \mathrm{p})$ a proton emiosion; the hatched region is interior to the sawtooth mixing radius, and the doubly hatched region is interice to the sawtooth inversion radius. The relative sizes of the latter two regions determine the characteristics of the proton sawtooth observed.

Fig. B.2 $15 \mathrm{MeV}$ proton signal from $\mathrm{d}\left({ }^{3} \mathrm{He}, \mathrm{p}\right) \propto$ fusion reactions (top trace), showing that tie proton signal can either decreane (a) or increase (b) at a sawtooth. (a) $B_{t}=31.8 \mathrm{kG} ; I_{p}=530 \mathrm{kA} ; T_{e 0}=2.3 \mathrm{keV}$; $\overline{n_{e}}=3.7 \times 10^{13} \mathrm{~cm}^{-3} ; P_{\mathrm{rf}}=2.6 \mathrm{MW} ;{ }^{3} \mathrm{He}$ concentration $=3.6 \% ; \mathrm{rt}_{*}=10 \mathrm{~cm}$. (b) $B_{t}=32.6 \mathrm{kG} ; I_{3}=590 \mathrm{kA} ; T_{\infty 0}=2.2 \mathrm{keV} ; \overrightarrow{n_{r}}=3.5 \times 10^{13} \mathrm{~cm}^{-3}$; $P_{\mathrm{ff}}=2.4 \mathrm{MW} ;{ }^{3} \mathrm{He}$ concentration $=2.9 \% ; r_{\mathrm{s}}=9 \mathrm{~cm}$.

Fig. B.3 Sowtootin oscillations in the d( $\left.{ }^{3} \mathrm{He}, \mathrm{p}\right) \alpha$ proton emisnion for a $2.3 \mathrm{MW}$ ICRH discizange on PLT with a $4 \%$-of-ne ${ }^{3} \mathrm{He}$ minority. Shot parameters are $B_{q}=2.6 \mathrm{kG}, I_{p}=570 \mathrm{kA}, \bar{n}_{q}=3.7 \times 10^{13} \mathrm{~cm}^{-3}$ average 
$T_{\infty 0}=2.0 \mathrm{keV}$, and neutron rate $I_{n}=1.4 \times 10^{12} / \mathrm{sec}$, implying an average $T_{\infty}$ of about $2.4 \mathrm{keV}$. Fast increases in proton emission are observed at each neutron sawtooth erast; the jumps are $5 \%$ in $30 \mu \mathrm{s}, 4 \%$ in $30 \mu \mathrm{s}$, and $4 \%$ in $50 \mu$ for the three everts shown. A detail of the second event is shown for clarity.

Fig. B.4 Change in the proton emission expected at a sawtooth. The horizontal axis is the savtooth inversion radius scaled by the plasma minor radius; the vertical axis is the minor radius of the resonance layer at the midplane, also scaled by the plasma minor radius. Calculations are for a source region $5 \mathrm{~cm}$ wide bounded by the resconance layer (Fig. B.1). The calculated change is never more than $5 \%$ for PLT conditions.

Fig. C.1 Lines of constant neutron emission $I_{n}$ and drop in emission $\Delta I_{n} / I_{n}$ in $T_{d b}$ and $\alpha_{T}$ space for a PLT case. A unique solution for $T_{d 0}$ and $a_{T}$ is assured. 


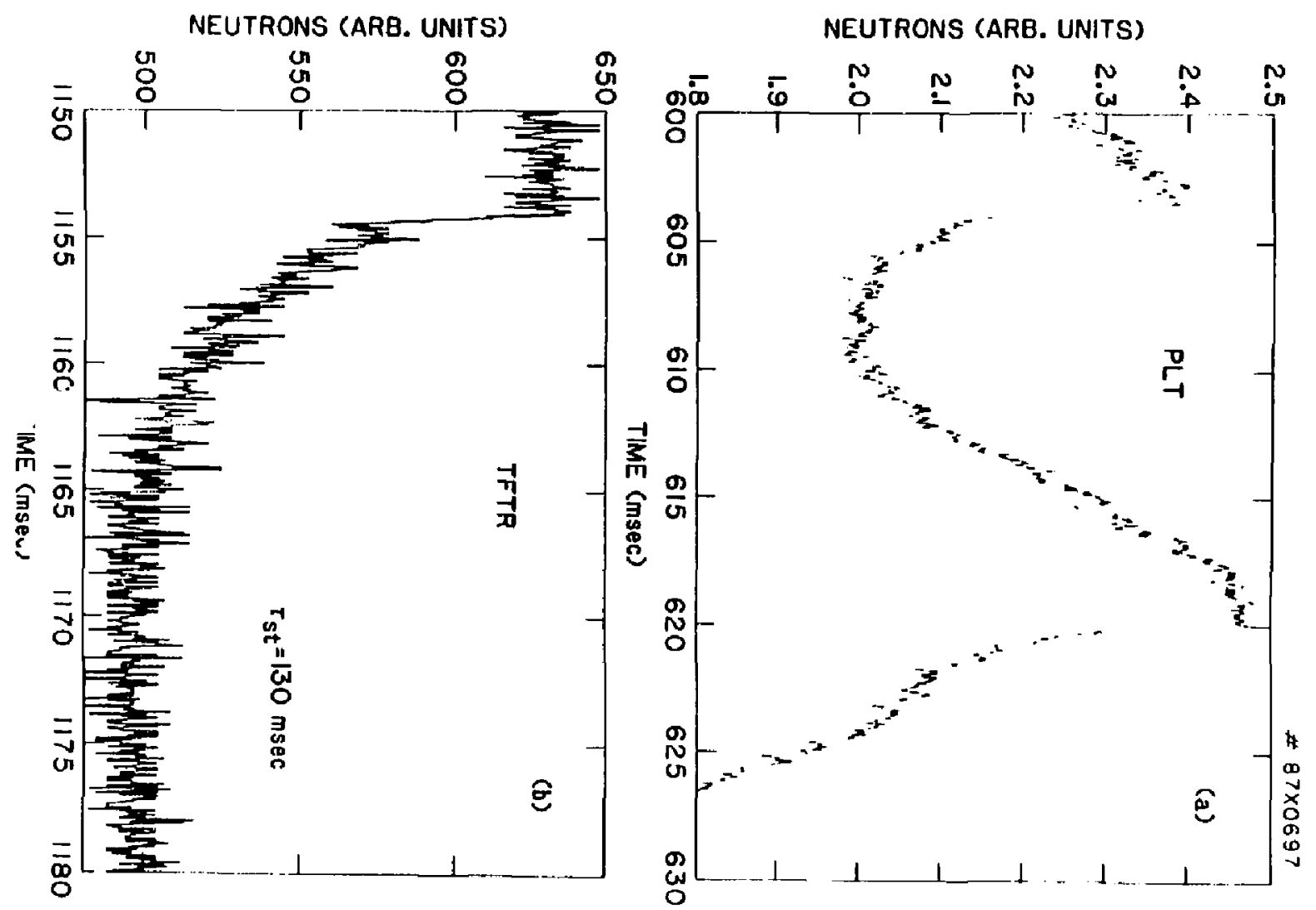




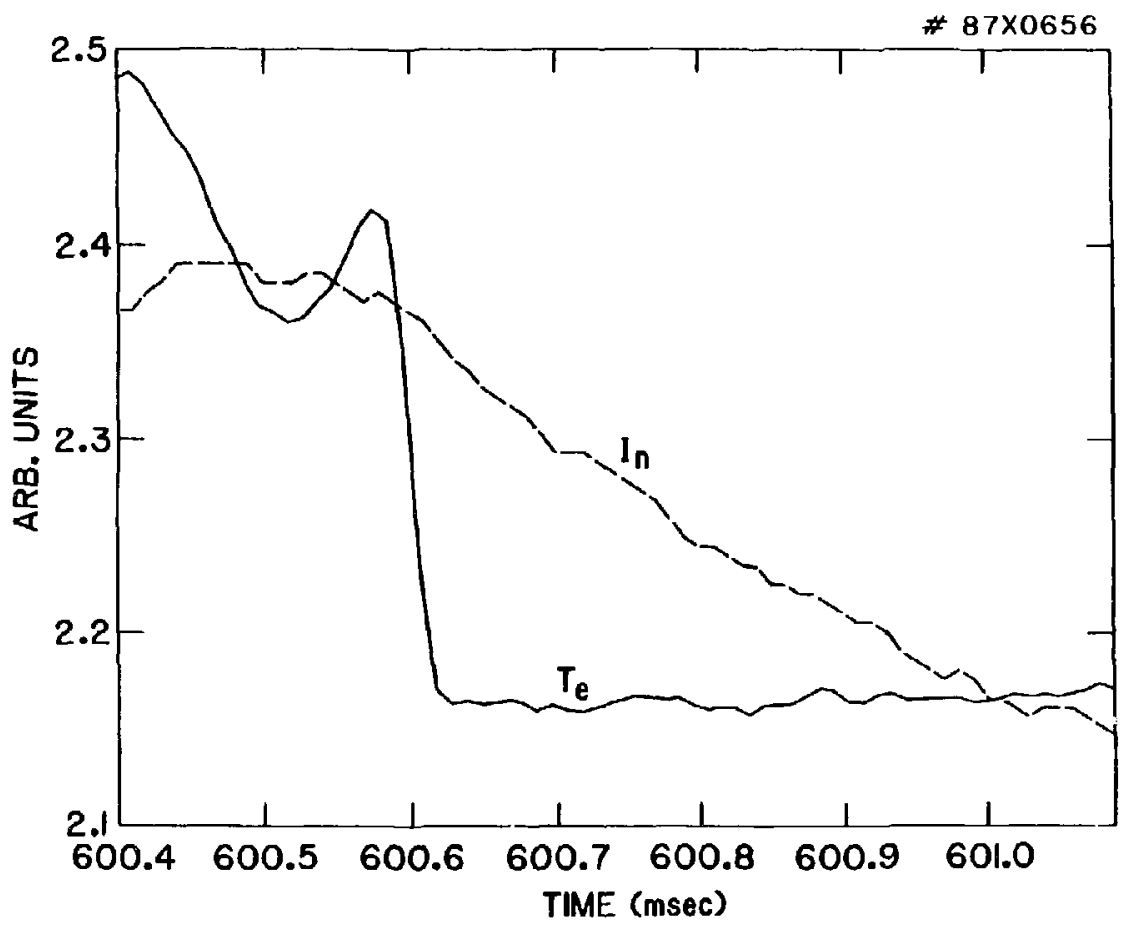

Fig. 2 
\# $87 \times 0812$

。

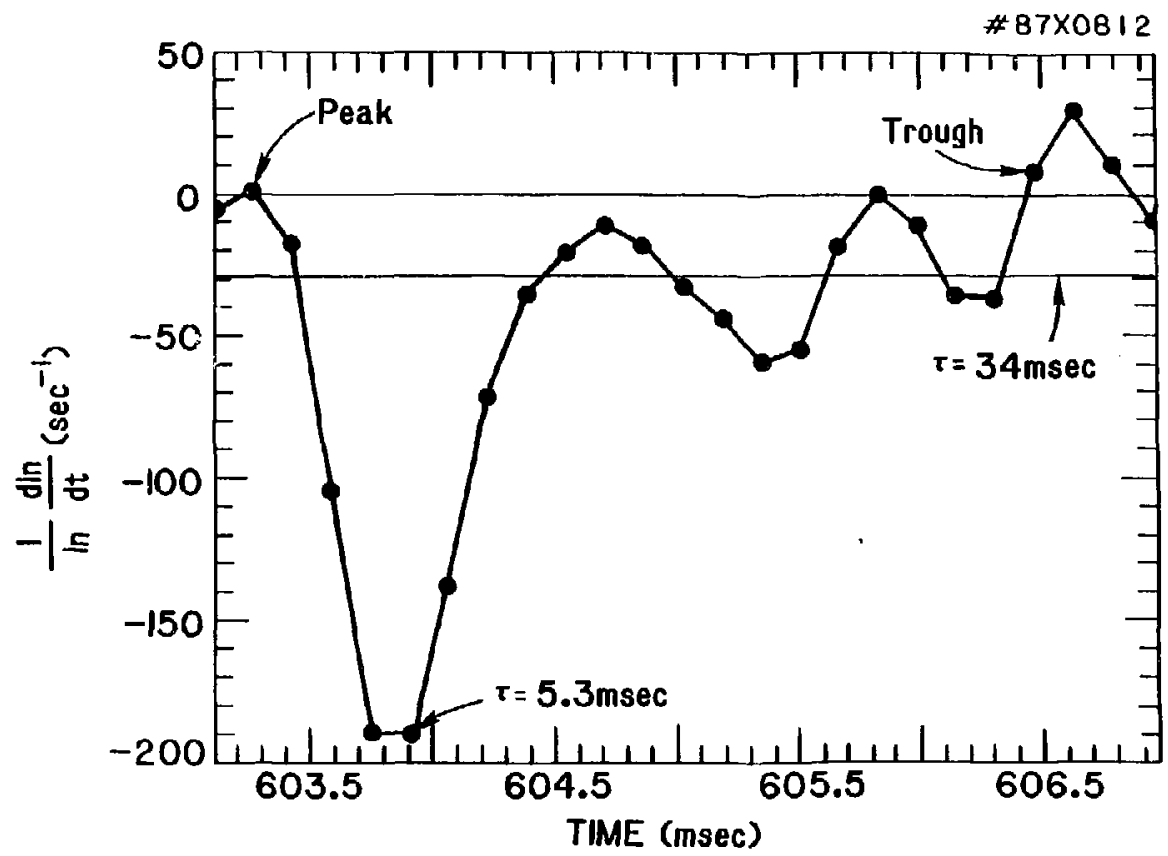

Eig. 3 


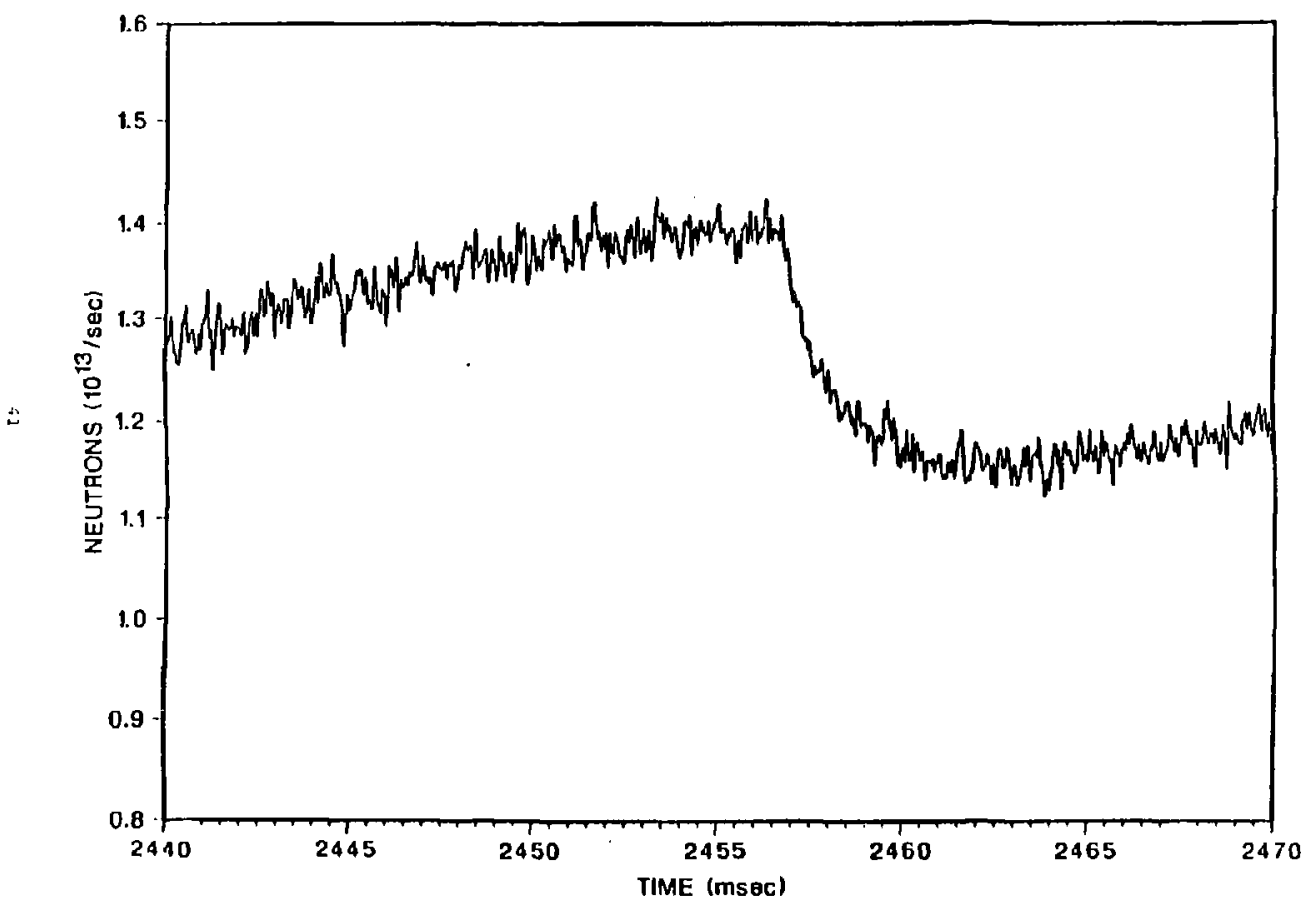




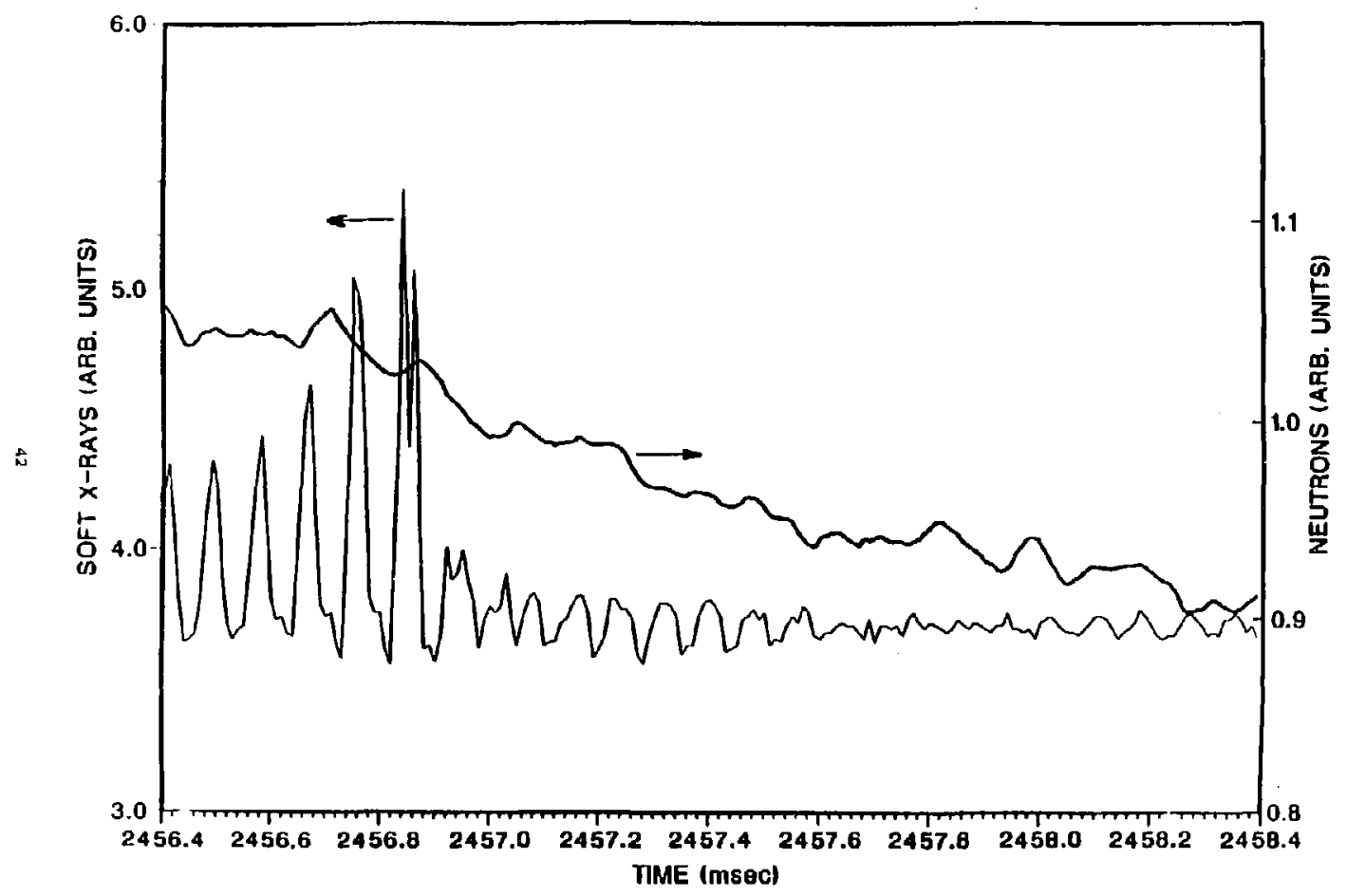

Fig. 5 


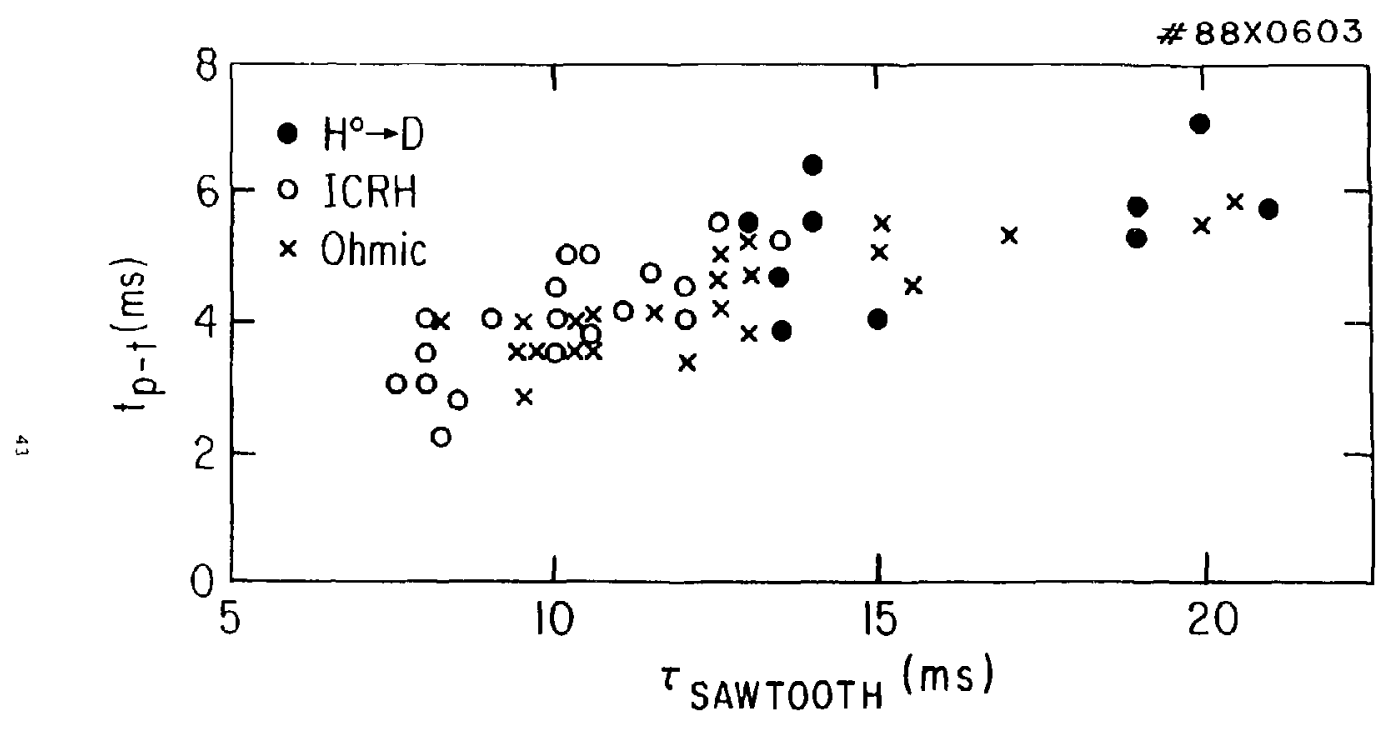




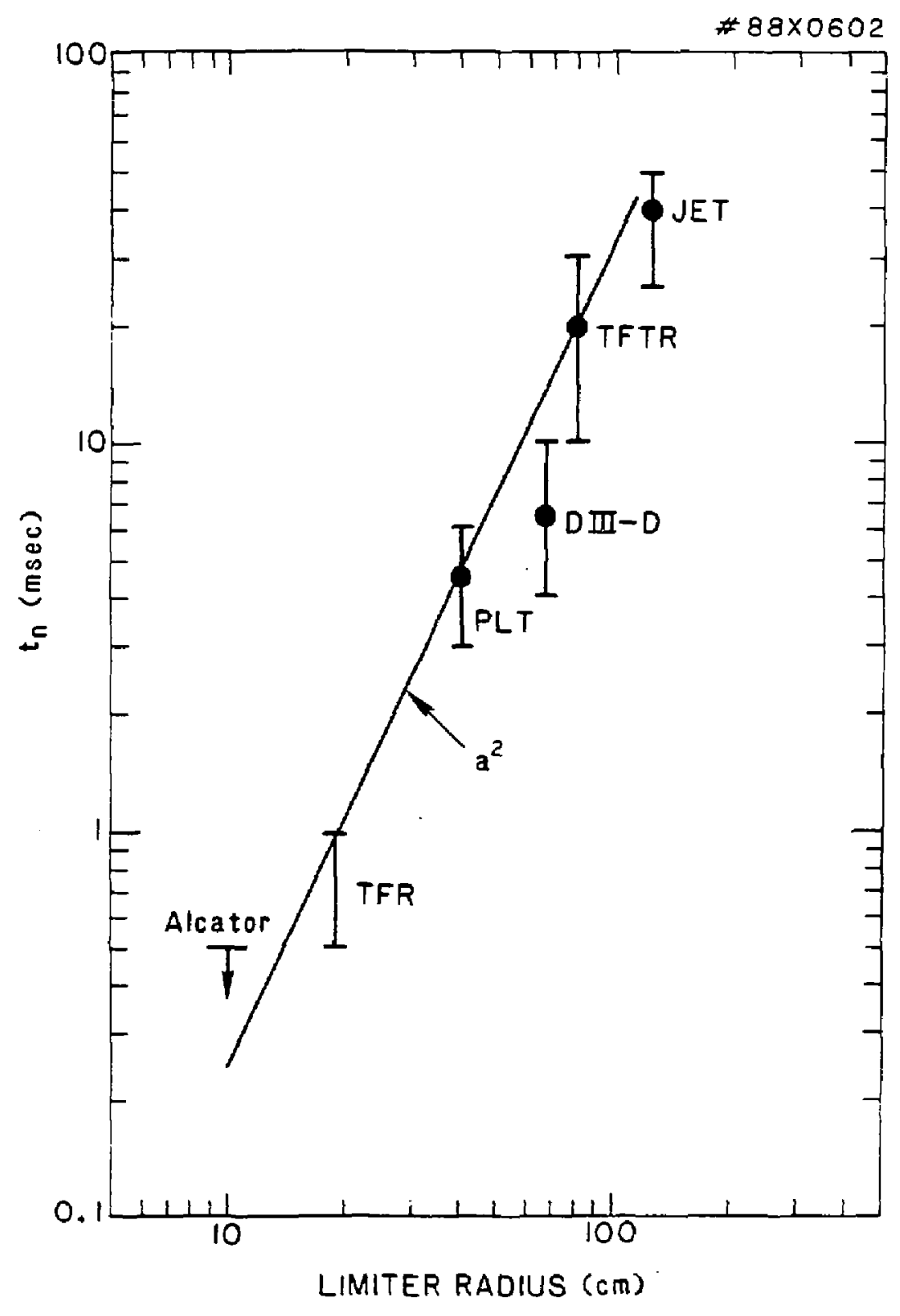

Eig. 7 


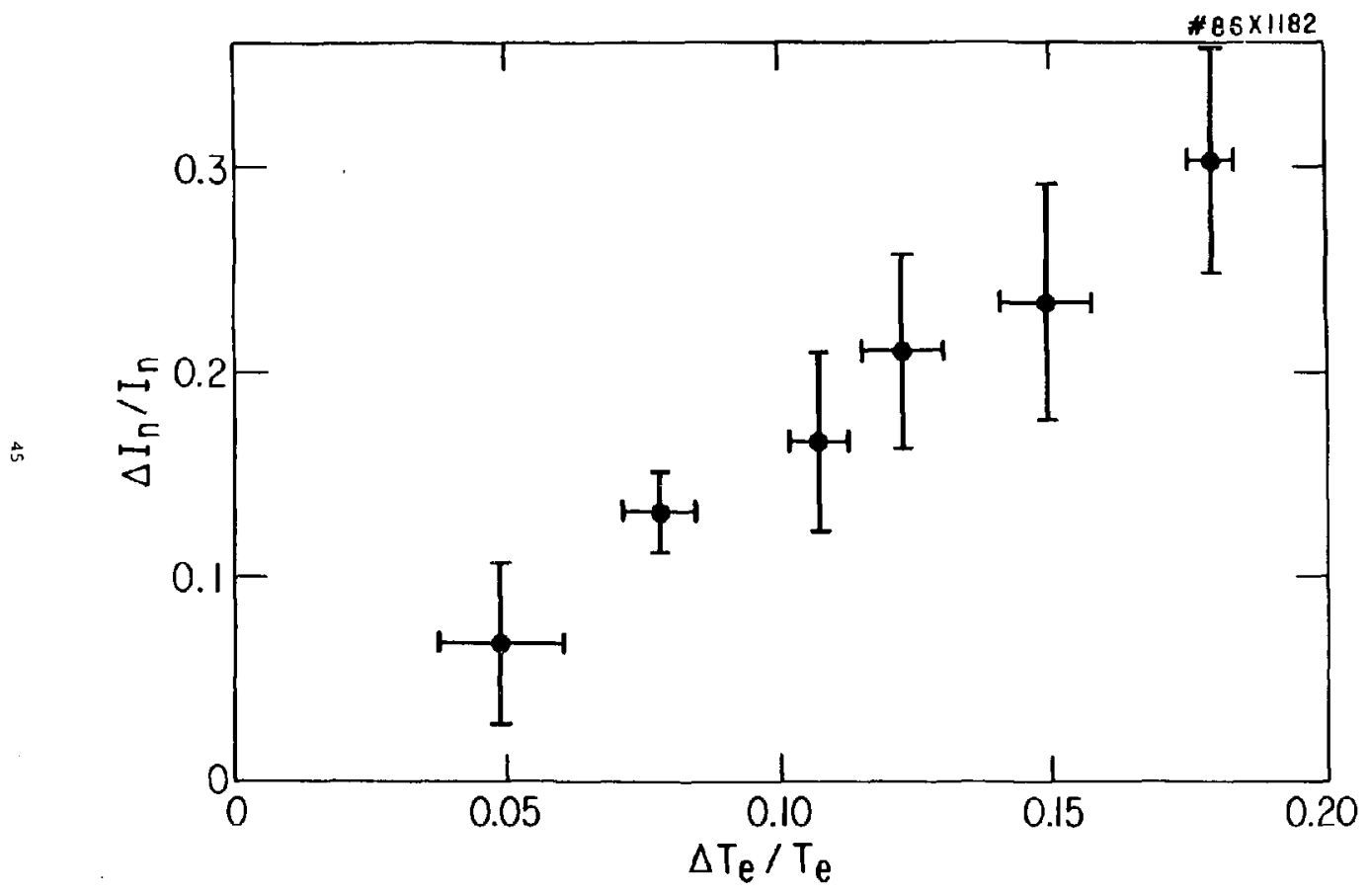

Fig. B 

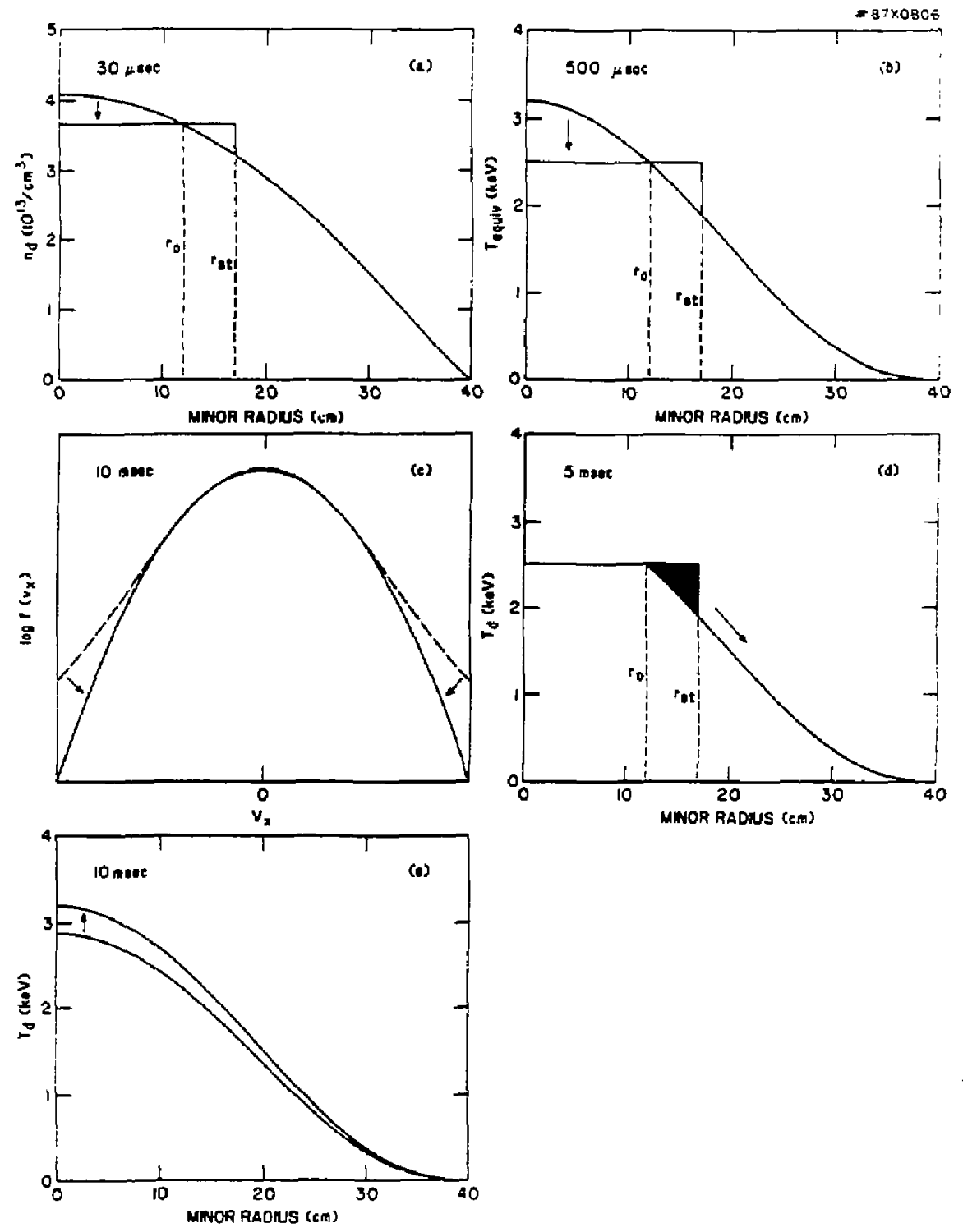

Fig. 9 


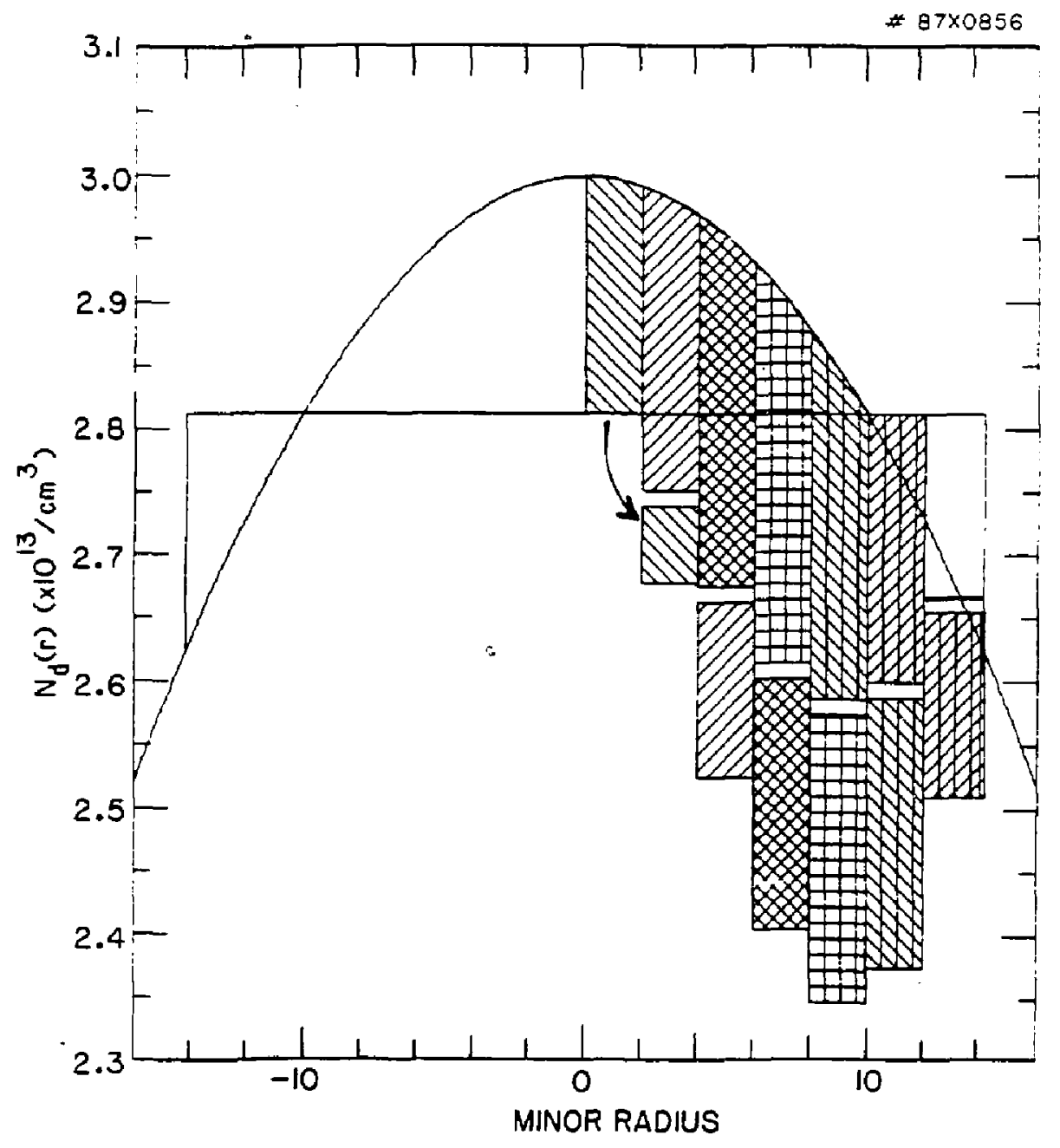

Eig. 10 


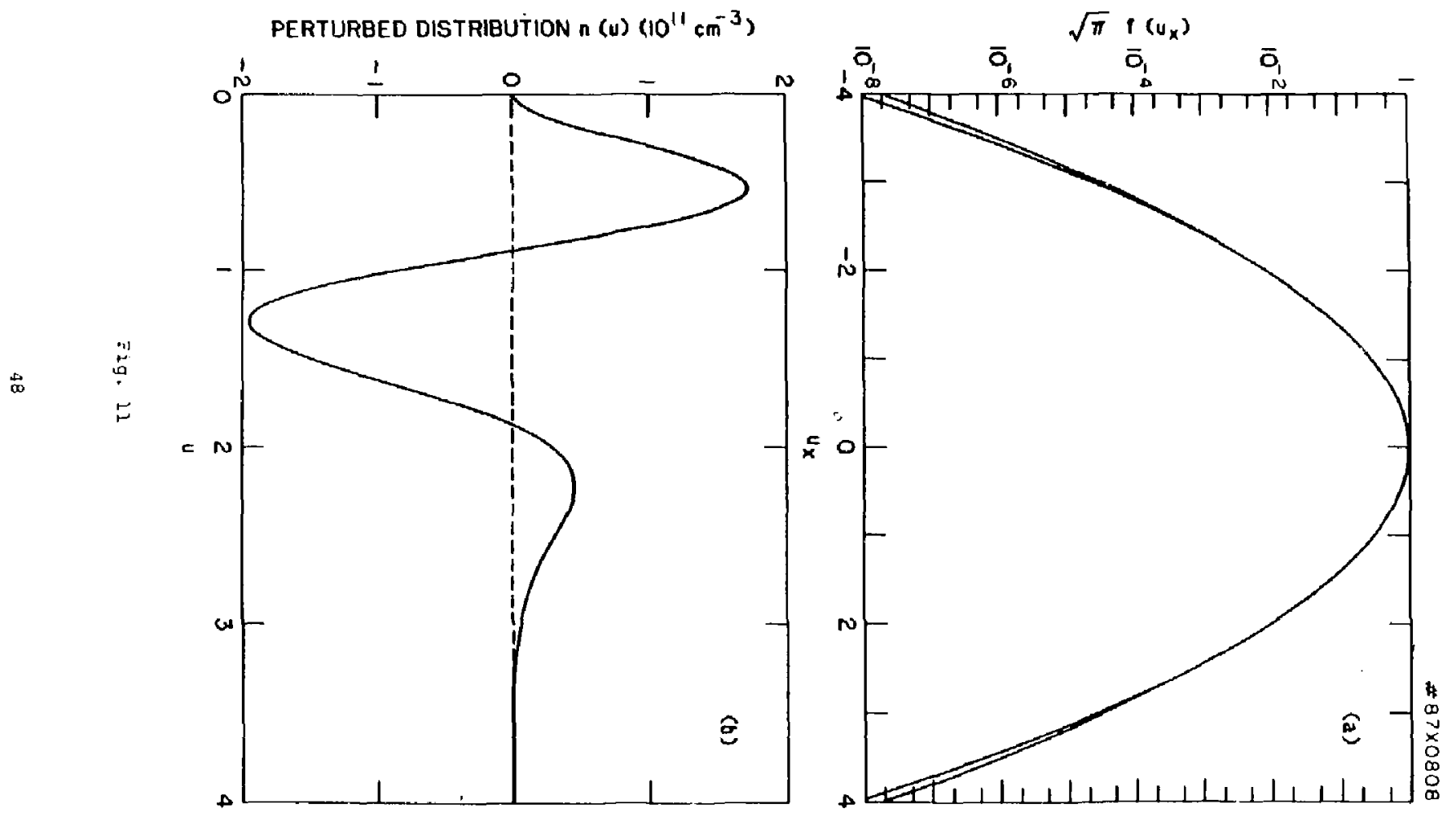




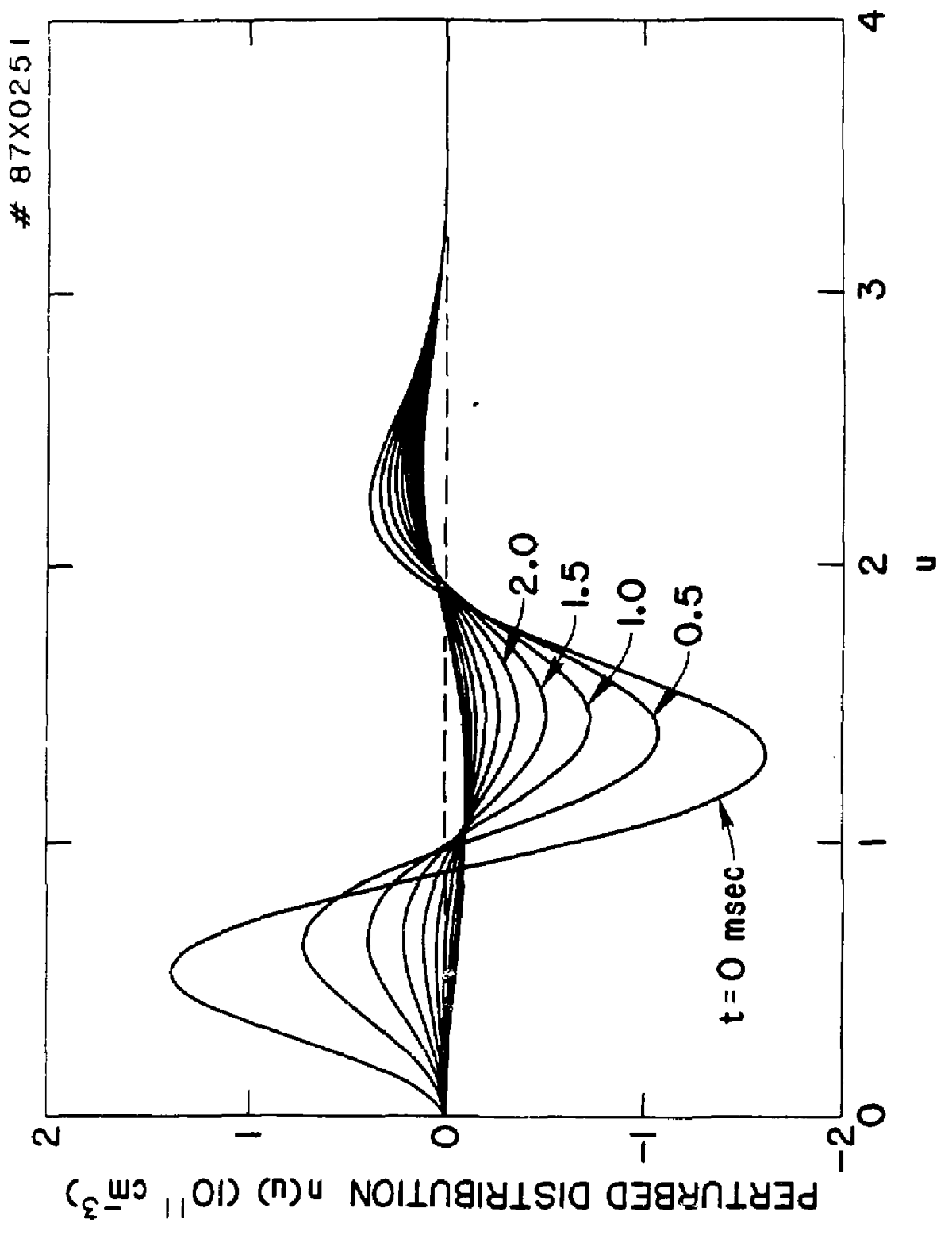

定 


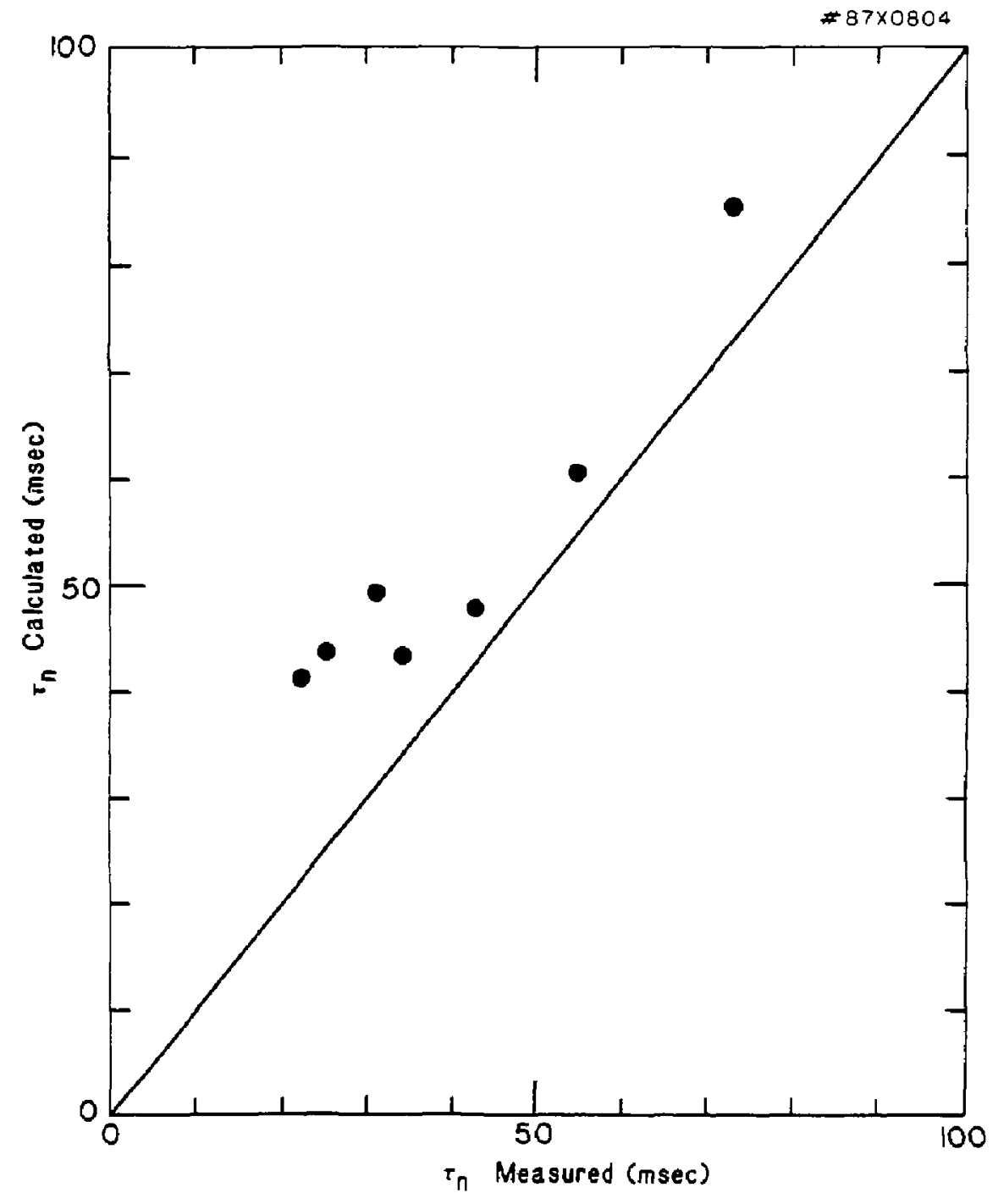

Fig. 13 

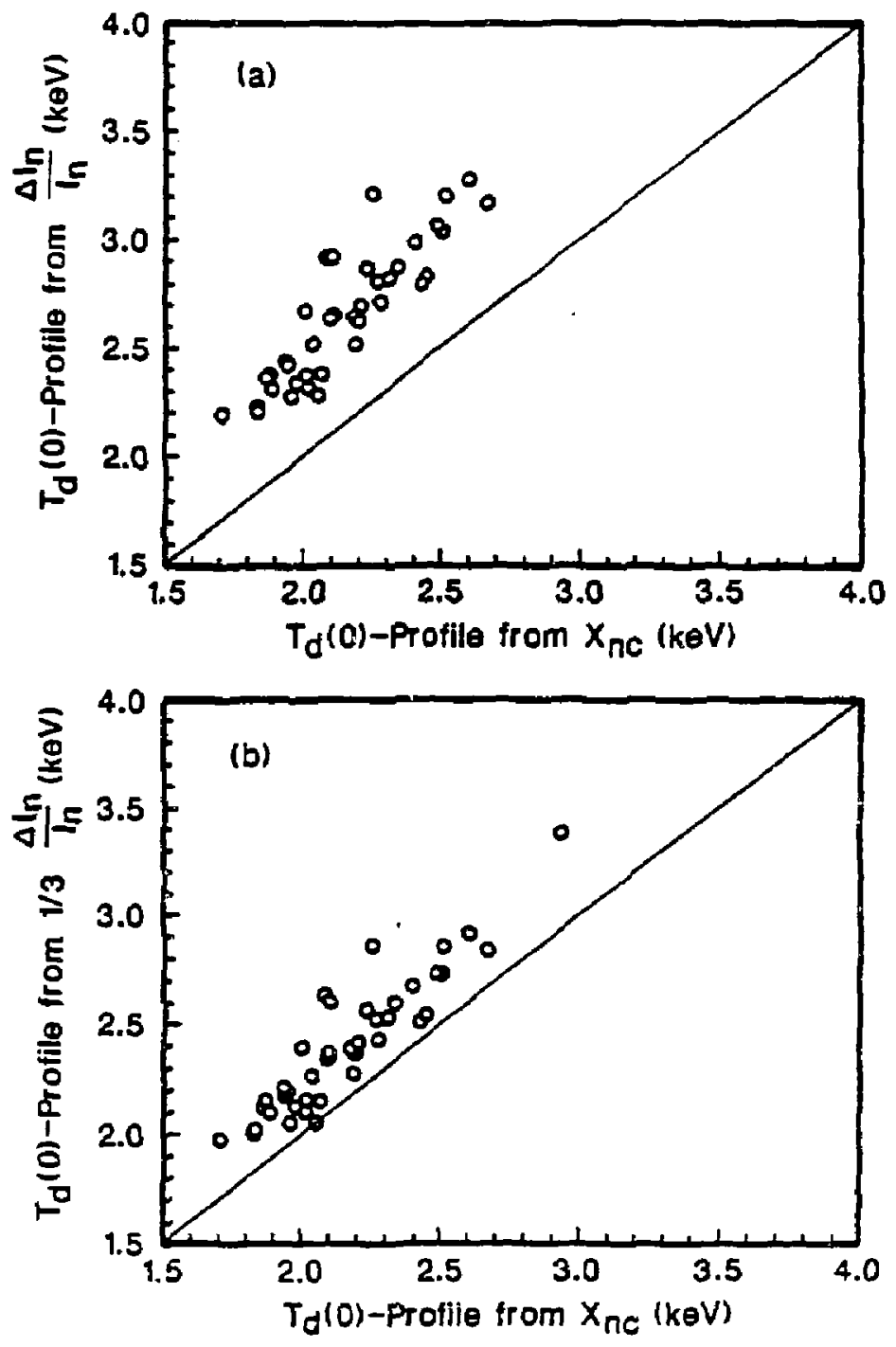

Fig. I4 


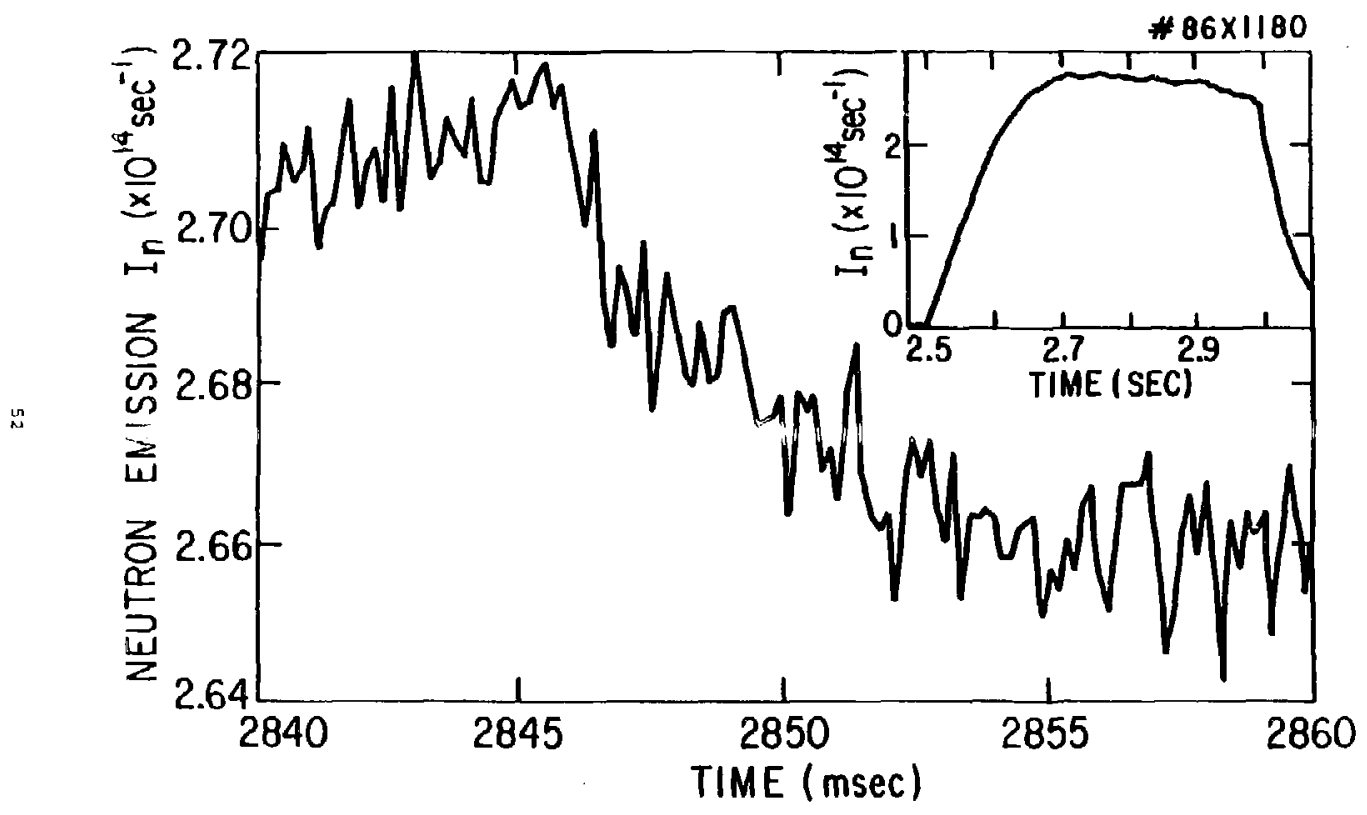




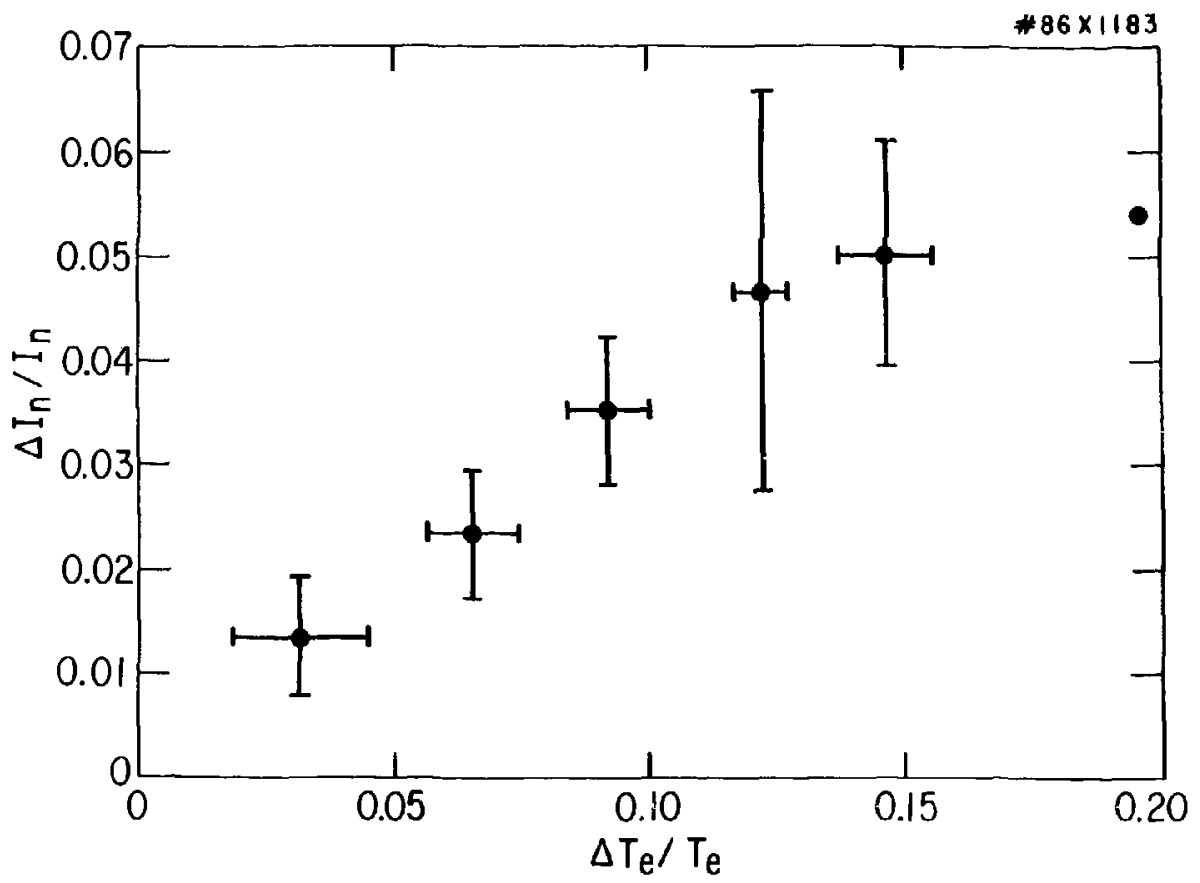




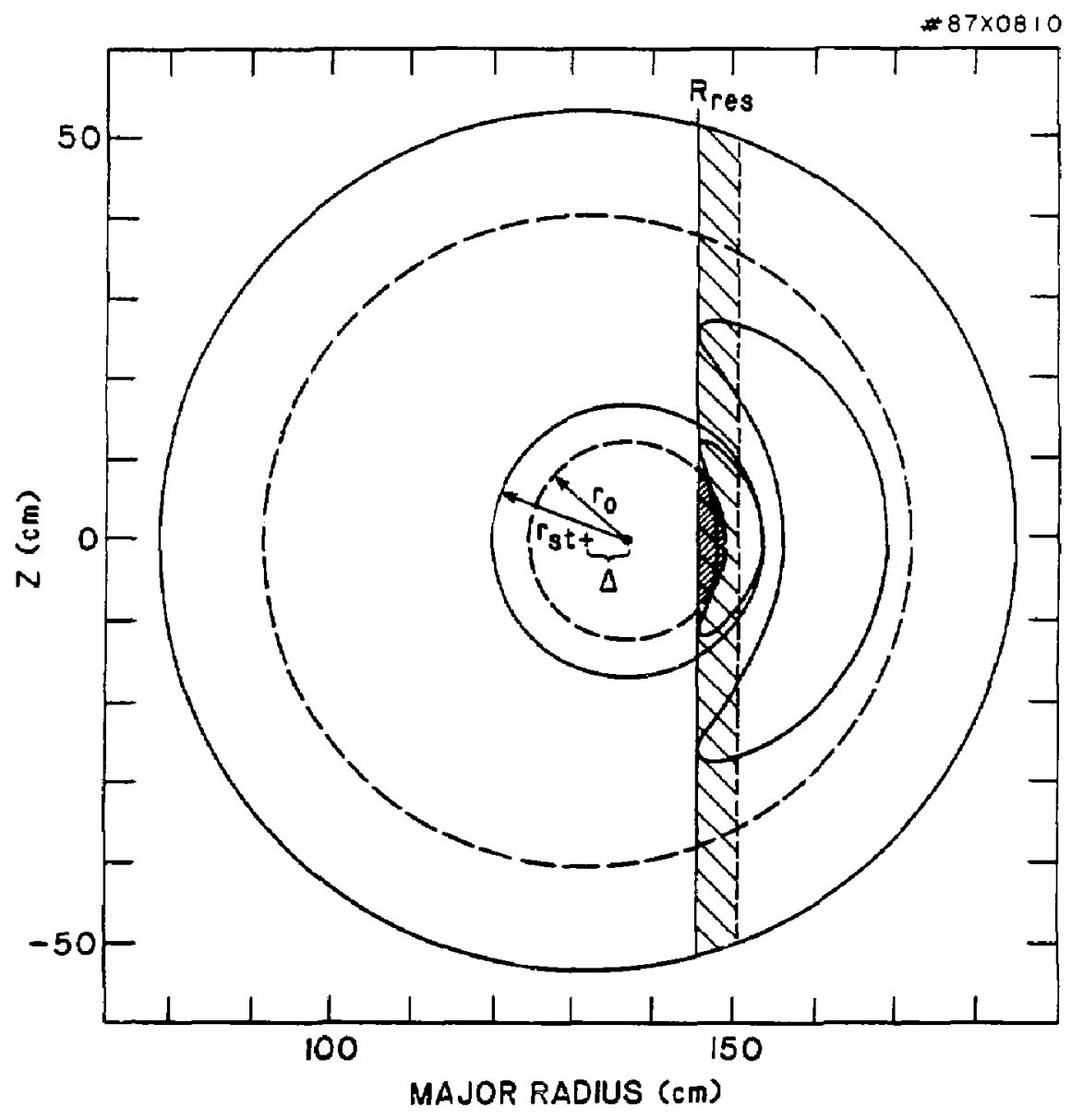

Fig. $B 1$ 

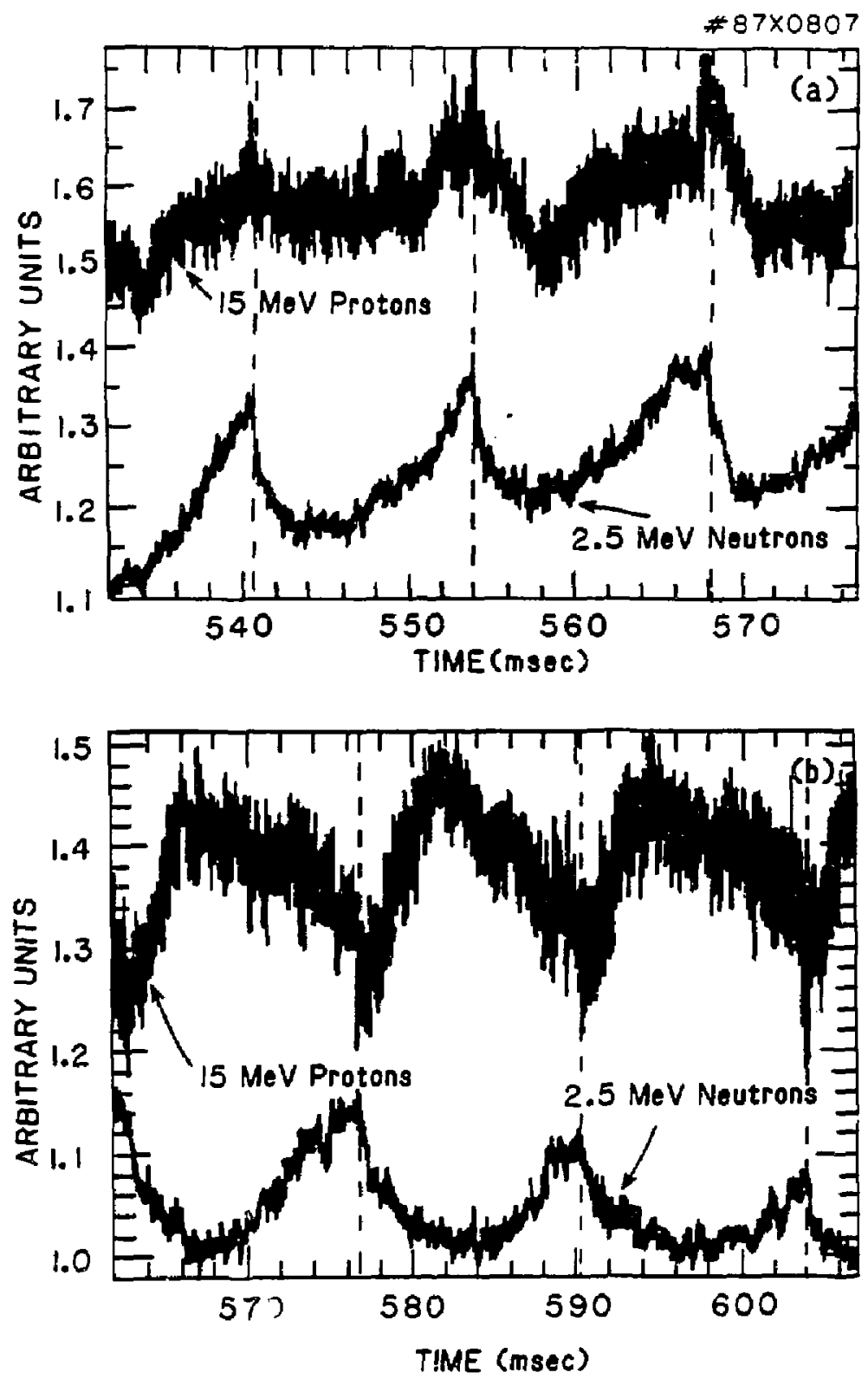

Fig. 82 


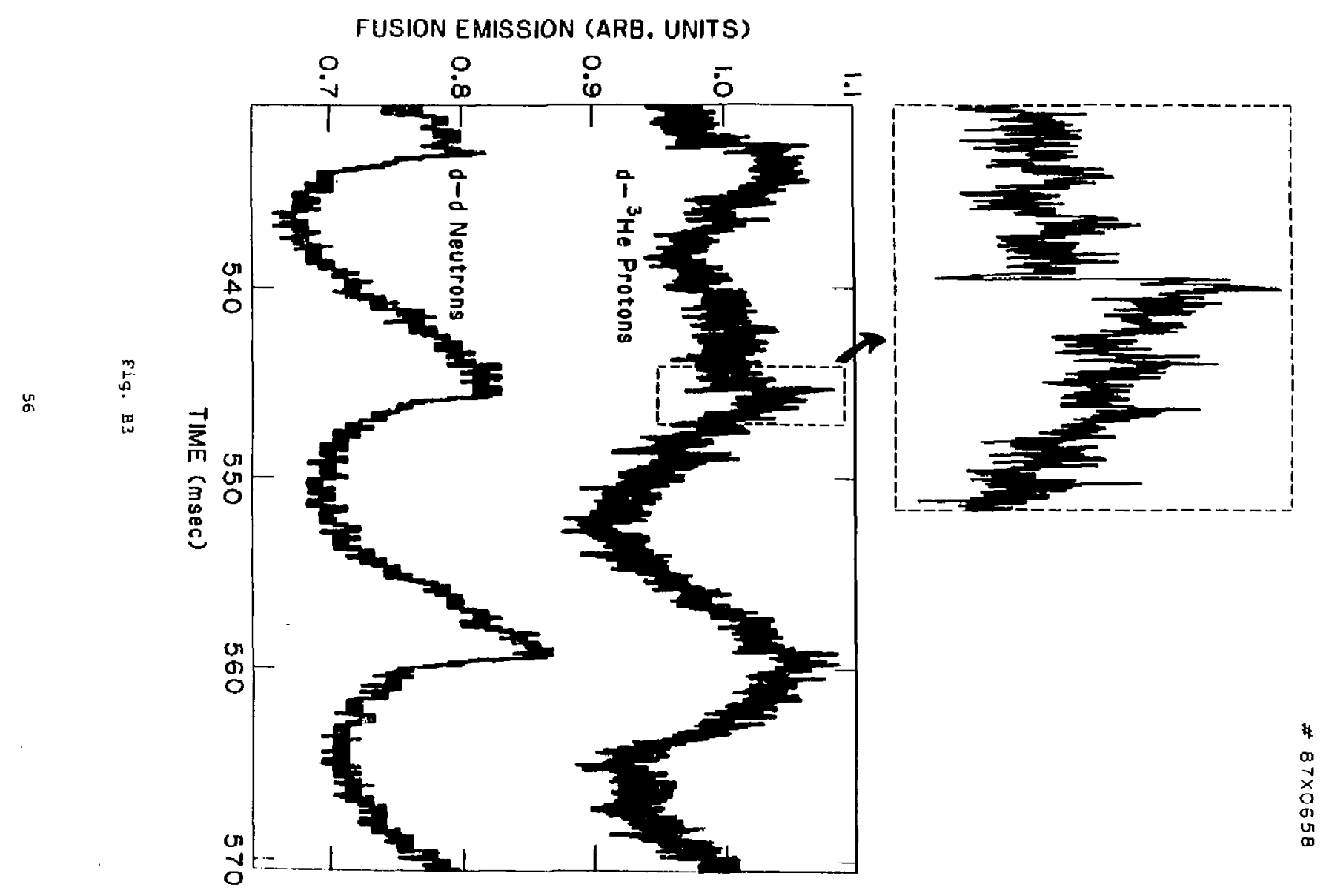




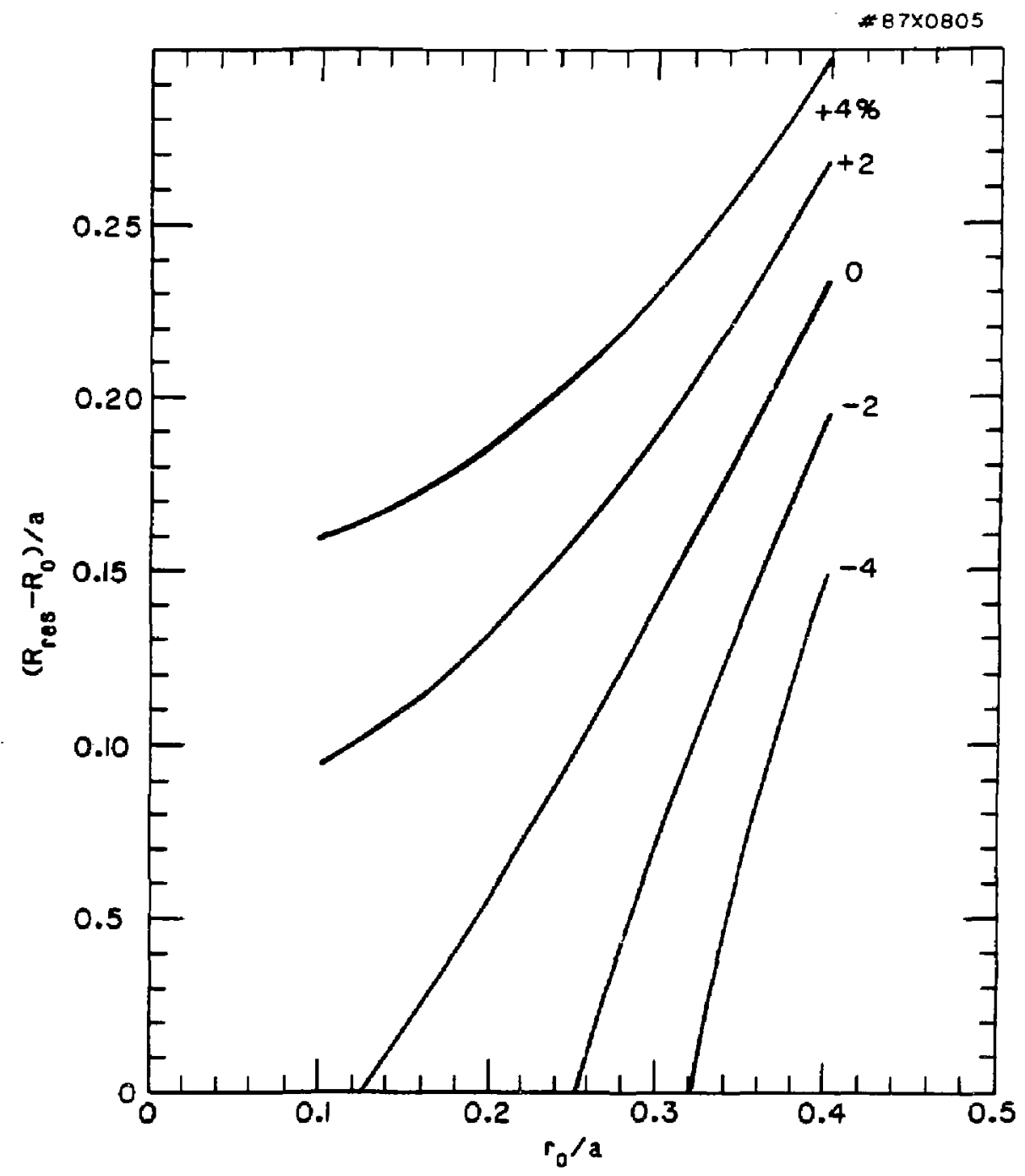

Fig. B4 


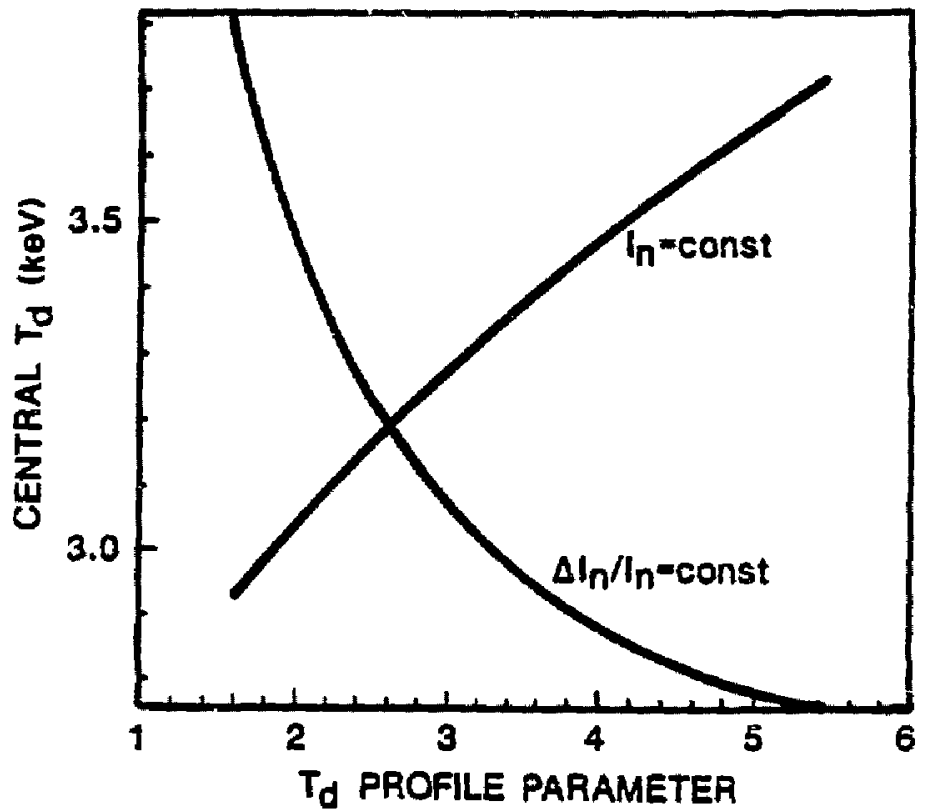

Fig. $\mathrm{Cl}$ 
Dr. Frank J. Paoloni, Univ of Wollongong, australia Prot, M.H, Brennen, Univ Sydney, AUSTRALIA Plasma Resaareh Lab., Australian Nat. Univ., AUstralia Prof. I,R. Jones, Flincers Univ., AUSTRALIA Prof. F. Cap, Inst Theo Phys, AuSTRIA

Prof. M. Haindior, Institut fur Theoret ische Physik, Austria M. Goossans, Astranomigeh Instituut, BELGIUm Ecole Rayale Militaire, Lab de Phys Plasnas, BELGIun Comission-Euroguan, $\alpha_{g}-x_{1} I$ fusion Prog, BELGIU Prof, R, Eouclique, Leboratoriun voor Natuurkunde, BELGIUA D. P.H. Sakanaka, Instituto Fisica, grazil Instituto On Pesquisas Esdeciasi-INFE, BRAZIL Documants office, Atcaic Energy of Canage Lielted, Cunpa Or. M.P. Bachynski, Mpe Technologies, Ine., Cawan Or. H,M. Skarsgard, University of Saskatehewan, Cawah Dr. H. Barnard, Univarsity of British Columia, Cavanan Prof. J. Teicheann, Univ, of Montraal, curad Prot. S.R. Sreanivasan, University of Calgary, CAwade Prof. Tudor W. Johnston, INes-Energle, CuraA Or. C.R. Jemes, Univ. of Alberte, CunaA Or. Poter Lukee, Konengkeno Univ, CZECroslovaKIA The Librarlan, Culhe Laboratory, EkELND The Librorlan, Autherford Applaton Lebordtory. ERTCNO Mrs. S.A. Hutchinson, JET Library, ENGLANO

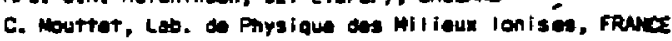
J. Redar, Cencronruate - Bat sos, FRunce

Univ. of loannins, Library of Physlics Dapt. EReECE Dr. Tou mal, Acadere Blbliograonic Sar. , hows kows

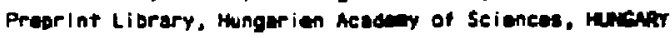
Or. B. Dasgudta, Sane linst of Mucl. Phys.. INDIA

Dr. P. Kas. Institute for Plases Rasearch, IROIA D. Ahllip Roenau, Isreal inst. Tech, ISRAEL Libra'tan, Int'I Ctr Theo Phy, ITALT Prof. G. Mutragni, Univ ol Padove, I TALY Miss Clel ia De inlo, Asece EuRaton-EkeA, I TALr giblioteca, Instituis dl fisica del pleses, Italy Dr. H. Yanato, Toshlibe Pre 2 . IAPNM

Prot. 1. Kawercel, Atole Energy Aas. Instltute, JAPN Prot. Kyoji Mlshikame, Univ of HIroshian. JPAN

Direc. Dept. Large Toksulk Res. JAERI, JAPAN

Prot. Setosti Itoh, Kyushy University. JAPAk

Researeh Info Center. Negove University, JAPNY

Prot. S. Tanake, Kyota University, JAPAN

Library. Kyoto Univarsity, JAPAM

Prot, Nobuyuki Inowe, University of Takyo, JAPAN

5. Mor I, JAERI, JNPAN

Librarian, Korea Advanced Energy Pas. Institute, KoReA

Prof. D.1. Choi, Adv. Inst Sel \& Tech, Kopen

Prol. B.S. LIley, University of Walkato, NEW ZEALANO

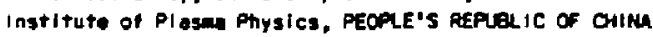

Lierarlan, Institute of Phys., PECPLE's REPU⿴囗十 IC Of OHINA Library. Tsing Hus univarsity. PEOPLE's REPUBLIC Of CHIM
2. Li, Southmest Inst. Physics, PECPLE'S REPUELIC Of CHINA Prof. J.A.C. Cabral, Inst Superior Tecnico, FORTUGAL

Or. Octovian Potrus, AL I CUzA Ĺniversity, ROMania Dr. Iahan de Villiars, Fugion Studies, AEC, SO AFRICA Prof. M.A. Hellogerg, University of Natal, SO AFRICA C.I.E.M.A.T., Fugion Div, Librory, SPAIN Or. Lennart Stentio, University of UAEA, SWEDEN Liorary, Royal inst Tech, SWEDEN

Prof. Mans Wlihelason, Cholmors Univ Tech, SWEDEN

Contre Phys des Plasaas, Ecole Polytech Fod, SWITZERLANo BIbliothonk, fom-lnst Voor PI agma-fysics, THE NETHERLANOS Dr. D.O. Ryutov, Sloerian Acad Sei, USSR

Or. G.A. Elisewr, Kurchator listl tute, USSR

Or. Y,A. Glukhikh, Inst Electrophysical ADparatus, USSR

Dr, Y,T. Tolok, Inst. Phys, Toch, USSR

Dr. L.M. Kovr Iznnykn, Institute Gen. Physics, USSR Nuelear Ras. Establishment, Jul ich Ltd., W. GERMANT Bibllothen, Inst. Fur Plasadorsehung, M. GERAANY Dr, K, Sehindler, Ruhr Universitat Bochun, W. GERMANY Asosx Rouding Ro, IPP/Man-Planck-Institut ur

Plaseaphrsik, W. Gefawy Librarian. Max-planek Institut, M. Gepawry Prof. R.K. Janev, Inat Phys, rucoslavia 